Notre Dame Journal of Formal Logic

Volume 47, Number 4, 2006

\title{
Hybrid Formulas and Elementarily Generated Modal Logics
}

\author{
lan Hodkinson
}

\begin{abstract}
We characterize the modal logics of elementary classes of Kripke frames as precisely those modal logics that are axiomatized by modal axioms synthesized in a certain effective way from "quasi-positive" sentences of hybrid logic. These are pure positive hybrid sentences with arbitrary existential and relativized universal quantification over nominals. The proof has three steps. The first step is to use the known result that the modal logic of any elementary class of Kripke frames is also the modal logic of the closure of this class under disjoint unions, generated subframes, bounded morphic images, and ultraroots. This latter class can be defined by the first-order sentences of a special syntactic form (called pseudo-equations by Goldblatt) that are valid in the former class. The second step is to translate these pseudo-equations into equivalent quasi-positive hybrid sentences. In the third and main step, we show that any quasi-positive sentence $S$ generates an infinite set of modal formulas called "approximants," which together axiomatize a canonical modal logic that is sound and complete for the class of frames validating $S$. The proof is analogous to standard proofs of Sahlqvist's theorem. It generalizes to sets of quasi-positive sentences. The main result now follows.
\end{abstract}

\section{Introduction}

We combine a Sahlqvist-like theorem with some translations into hybrid logic in order to axiomatize all and only the modal logics of elementary classes of frames. (In this paper, a class is elementary if it is the class of models of a possibly infinite set of first-order sentences.) In the introduction, we outline the work and its context.

1.1 Elementarily generated modal logics A modal logic is said to be canonical if it is valid in the frame of its own canonical model. The canonical model construction

Received April 7, 2006; accepted June 25, 2006; printed December 28, 2006 2000 Mathematics Subject Classification: Primary, 03B45

Keywords: canonical modal logic, elementary frame class, Sahlqvist theorem, hybrid logic

(C)2006 University of Notre Dame 
involves forming the Kripke frame whose worlds are the maximal consistent sets and with a "canonical" accessibility relation and assignment (or valuation). It came to prominence through the work of Lemmon and Scott [30] and also (independently) Cresswell [6] and Makinson [31], although Jónsson and Tarski [26] had introduced a similar construction earlier. Any canonical logic is the logic of its own canonical frame. (The logic of a Kripke frame $\mathcal{F}$, or a class $\mathcal{K}$ of Kripke frames, is the set of modal formulas that are valid in $\mathcal{F}$ or, respectively, in every frame in $\mathcal{K}$.) So showing a logic to be canonical yields up a "free" completeness theorem for it and has proved to be a useful and widely applicable method of proving completeness theorems for modal logics.

It turned out that all the canonical modal logics seen in practice were the logics of elementary classes of frames. We call such logics elementarily generated. A seminal result of Fine [7] showed that this was no accident: any elementarily generated modal logic is canonical. ${ }^{1}$ The converse is not true in general ([17], [16]), and this raises the problem of characterizing both the canonical and the elementarily generated logics in some independent way in order to understand them and their similarities and differences better. It seems that all known "natural" examples of canonical logics are elementarily generated, so certainly the elementarily generated logics are worthy of attention.

One kind of characterization that would be attractive is a syntactic one. An elementarily generated logic may be presented to us syntactically by giving its axioms, in which case it may not be evident that the logic is elementarily generated, or semantically by giving an elementary class of frames that determines the logic, in which case we may not have modal axioms for it. We would like

(A) to be able to tell when a given set of modal axioms determines an elementarily generated logic,

(B) to generate explicit axioms in some effective way for any semantically-given, elementarily generated logic.

1.2 Sahlqvist's theorem One obvious paradigm for such results is Sahlqvist's theorem [32]. There, a syntactic blueprint is given for generating modal formulas called Sahlqvist formulas. Positive formulas had been examined in this context much earlier [26], and Sahlqvist formulas can perhaps be thought of as generalized positive formulas. See Example 3.6 for a definition. Common examples include $\square p \rightarrow p$, $\square p \rightarrow \square \square p$, and $\diamond \square p \rightarrow \square \diamond p$.

Sahlqvist's theorem addresses problem (A) above. Every Sahlqvist formula $\sigma$ axiomatizes an elementarily generated logic. It is the logic of an elementary class of Kripke frames that is definable by a single first-order sentence, the so-called firstorder correspondent of $\sigma$. The correspondent is obtainable from $\sigma$ by a simple algorithm. For example, the correspondent of $\square p \rightarrow p$ is $\forall x R(x, x)$, where $R$ is the accessibility relation of the frame.

Sahlqvist-axiomatizable logics are well behaved in other ways. A single modal logic can be determined by several different classes of frames. A frame for a logic is a (Kripke) frame that validates every formula of the logic. Now it is possible for the class of all frames for an elementarily generated logic to be nonelementary; we will see examples below. But this cannot happen for Sahlqvist-axiomatizable logics: we get the "Sahlqvist bonus" that the correspondent of $\sigma$ is valid in precisely the frames 
validating $\sigma$-and, as can be seen either by Fine's theorem or directly, these include the canonical frame of the logic.

There is even a converse result. In [28], §5.6, Kracht identified a fragment of first-order logic (in the signature of frames) that contains the correspondent of every Sahlqvist formula. Given any sentence $\chi$ in the fragment, he showed how to synthesize a Sahlqvist formula whose correspondent is equivalent to $\chi$; this addresses problem (B).

1.3 Limitations of Sahlqvist's theorem Sahlqvist's theorem has been immensely useful in practice since it is easy to use and covers a wide range of examples. It has been generalized by several authors-for example, [34], [18], [19], [27], and [35]. However, it does not fully answer our problems (A) and (B), because it does not cover all elementarily generated logics.

We will give three kinds of examples of this. The first involves McKinsey's formula $M=\square \diamond q \rightarrow \diamond \square q$. The logic axiomatized by $M$ alone is not elementarily generated, but the logic $K 4.1$ axiomatized by $M$ together with the transitivity axiom $\square q \rightarrow \square \square q$ is elementarily generated: these axioms are valid precisely in the transitive frames satisfying

$$
\forall x \exists y(R(x, y) \wedge \forall z t(R(y, z) \wedge R(y, t) \rightarrow z=t)),
$$

and so $K 4.1$ is the logic of this elementary class of frames. It is well known that $K 4.1$ is not Sahlqvist axiomatizable. (For details, see, e.g., [3], p. $168 .^{2}$ ) It is an example of a cofinal subframe logic. Very powerful techniques to handle these logics are available, and consequently their properties are quite well understood. For example, all canonical cofinal subframe logics are elementarily generated. Modal axioms can be synthesized for any cofinal subframe logic [4]; this is along the lines of (B) above, but it does not cover all elementarily generated logics and covers some nonelementarily generated ones, so its scope is rather different from our concerns here.

There is a wider class of elementarily generated logics not covered by Sahlqvist's theorem. Already in [30], Lemmon generalized McKinsey's formula $M$ to a whole sequence of formulas

$$
M_{n}=\diamond\left(\left(\diamond q_{1} \rightarrow \square q_{1}\right) \wedge \cdots \wedge\left(\diamond q_{n} \rightarrow \square q_{n}\right)\right) \quad(n \geq 1) .
$$

$M_{1}$ is equivalent (in the basic modal logic $K$ ) to $M$, and in the presence of the transitivity axiom, all the $M_{n}$ follow from $M_{1}$. The logic $K M^{\infty}$ axiomatized by (2) is the logic of the class of (possibly intransitive) frames defined by (1). It is therefore elementarily generated, and canonical. Indeed, (1) is true in its canonical frame, though it is not true in all frames for $K M^{\infty}$ : we lose half of the "Sahlqvist bonus" referred to above. But $K M^{\infty}$ cannot be axiomatized by any set of (even generalized) Sahlqvist formulas, because the class of frames for $K M^{\infty}$ is nonelementary ([15], [2]), and any axiomatization of it must involve infinitely many noncanonical formulas [15]. (A formula is canonical if it axiomatizes a canonical logic. All Sahlqvist formulas are canonical.)

The same is true for the logic $K M T$ studied by Hughes [24], characterized by the class of frames satisfying

$$
\forall x \exists y(R(x, y) \wedge R(y, y)),
$$


and axiomatized by

$$
M T_{n}=\diamond\left(\left(\square q_{1} \rightarrow q_{1}\right) \wedge \cdots \wedge\left(\square q_{n} \rightarrow q_{n}\right)\right) \quad(n \geq 1) .
$$

A related example can be found in [23]. If we are willing to move to an algebraic setting, the variety RRA of representable relation algebras is another example [23]. All these logics are canonical and indeed elementarily generated. However, not only are they not finitely axiomatizable and not Sahlqvist axiomatizable, but any axiomatization requires infinitely many noncanonical formulas. Canonicity emerges only when all the axioms are taken together. It seemed that this rather striking phenomenon of "canonicity in the limit" was a new source of canonicity beyond the scope of Sahlqvist's theorem, and it needed an explanation.

A partial explanation was provided by Balbiani et al. in [2], where it was shown that for any Sahlqvist formula ${ }^{3} \varphi\left(p_{1}, \ldots, p_{n}\right)$ with local first-order correspondent $\sigma(x)$, the logic axiomatized by

$$
\left\{\diamond\left(\varphi\left(p_{1}^{1}, \ldots, p_{n}^{1}\right) \wedge \cdots \wedge \varphi\left(p_{1}^{m}, \ldots, p_{n}^{m}\right)\right): m \geq 1\right\},
$$

where the $p_{j}^{i}$ are distinct atoms, is canonical and is the logic of the class of frames defined by $\forall x \exists y(R(x, y) \wedge \sigma(y))$. This Sahlqvist-like result covers $K M^{\infty}$ and $K M T$, as is evident from (2) and (4). The full scope of the method is unclear. It remains to be seen whether it can be generalized to cover all elementarily generated logics. [2] lists some interesting open problems to do with it.

One more example of an elementarily generated modal logic not covered by Sahlqvist's theorem involves additivity axioms. In [7], Fine showed that

$$
\diamond \square p \rightarrow \diamond \square(p \wedge q) \vee \diamond \square(p \wedge \neg q)
$$

is canonical and axiomatizes the logic of the class of frames satisfying

$$
\forall x y(R(x, y) \rightarrow \exists z(R(x, z) \wedge \forall u v(R(z, u) \wedge R(z, v) \rightarrow u=v \wedge R(y, v)))) .
$$

However, the class of all frames for (5) is nonelementary, so (5) cannot be replaced by Sahlqvist axioms. The study was extended by Jónsson and Venema ([25], [39]) to general additivity axioms

$$
\pi(p \vee q) \rightarrow \pi(p) \vee \pi(q)
$$

for positive $\pi(x)$. (An axiom equivalent to (5) is obtained by taking $\pi(x)=\diamond \square x$ in (6).) Venema proved that (6) axiomatizes the logic of an elementary class $\mathcal{K}_{\pi}$ of frames and showed how to construct a single first-order sentence $\sigma_{\pi}$ defining $\mathcal{K}_{\pi}$ : cf. problem (A) above. He called $\sigma_{\pi}$ and (6) canonical pseudo-correspondents, since (6) is valid in any frame satisfying $\sigma_{\pi}$, and the canonical frame of the logic axiomatized by (6) satisfies $\sigma_{\pi}$. For some $\pi$ (e.g., $\square \diamond x$; see Example 4.7 below), (6) axiomatizes a Sahlqvist logic; for other $\pi$, it does not. Venema states in [39] that his original motivation was to "axiomatize [the modal logic of] an elementary frame class": cf. problem (B) above.

1.4 Our results In this paper, we will prove a Sahlqvist-like theorem that allows us to axiomatize all elementarily generated modal logics. We show that a modal logic is elementarily generated precisely when it is axiomatizable by modal axioms of a particular syntactic form. Starting with an elementary class $\mathcal{K}$ of frames axiomatized by a first-order theory $T$, the method applies three steps: 
1. Form the theory $U$ consisting of the first-order sentences which are consequences of $T$ and have (roughly) the form $\forall x \psi(x)$, where $\psi(x)$ is built from atomic formulas using $\wedge, \vee$, and relativized quantifiers

$\forall y_{1}, \ldots, y_{k}\left(R\left(x, y_{1}, \ldots, y_{k}\right) \rightarrow \psi\right)$ and $\exists y_{1}, \ldots, y_{k}\left(R\left(x, y_{1}, \ldots, y_{k}\right) \wedge \psi\right)$.

Sentences of this form have been studied by, for example, Goldblatt and van Benthem, and it is known that $U$ axiomatizes the class $\overline{\mathcal{K}}$ of frames obtained by closing $\mathcal{K}$ under bounded morphic images, generated subframes, disjoint unions, and ultraroots, and that the modal logics of $\mathcal{K}$ and $\overline{\mathcal{K}}$ are the same.

2. Translate each first-order sentence $\sigma$ in $U$ into a "pure quasi-positive sentence" of hybrid logic that is valid in precisely the models of $\sigma$. This can be done by a simple algorithm. Let $\Phi$ be the resulting set of hybrid sentences. $\overline{\mathcal{K}}$ is the class of frames in which all sentences in $\Phi$ are valid.

3. Translate each sentence in $\Phi$ into an infinite set of modal formulas. (Again, this can be done by a simple algorithm.) The modal logic $\Lambda_{\Phi}$ axiomatized by them is the logic of $\mathcal{K}$ (and of $\overline{\mathcal{K}}$ ).

Since pure hybrid sentences define elementary classes, all and only the elementarily generated modal logics have the form $\Lambda_{\Phi}$ for a set $\Phi$ of pure quasi-positive hybrid sentences. In this way, we are able to enumerate modal axioms for every elementarily generated modal logic, as per problem (B) above. $\Phi$ can be regarded as a canonical pseudo-correspondent of $\Lambda_{\Phi}$ in the sense of [39] and as discussed above.

Let us discuss the three steps in more detail. Step 1 is straightforward, using firstorder proof theory. In step 2, we translate the first-order sentences from step 1 into pure quasi-positive hybrid sentences. Hybrid formulas involve nominals: special propositional atoms that are always assigned to singleton subsets of a Kripke frame and serve as names for worlds. The quasi-positive hybrid formulas allow arbitrary existential and relativized universal quantification over nominals, this last being the only place that negation can be used. A pure formula involves no propositional atoms; in a sentence, all nominals are bound by quantifiers. The translation into quasi-positive hybrid sentences is fairly straightforward, and the reader may wonder why we bother with it. The answer is that quasi-positive sentences are modal-like formulas to which step 3 can be applied.

The modal axioms obtained in step 3 are generated as "approximants" to the formulas in $\Phi$. In any Kripke model, we may approximate a hybrid formula $\varphi$ by taking a finite partition of the frame induced by the truth values of a finite set $S$ of modal formulas (as in filtration), assigning nominals to partition classes and simulating existential and universal quantification of nominals by (finite) disjunction and conjunction over partition classes, respectively. This can be written out as a modal formula, called an approximant of $\varphi$. It is explicitly constructible from $\varphi$ and $S$, if $\varphi$ is a sentence.

It turns out that if a quasi-positive sentence $\varphi$ is valid in a frame, then so are all its approximants. Conversely, as the set $S$ increases, the approximants simulate $\varphi$ more and more closely. In the canonical model of the logic $\Lambda_{\varphi}$ axiomatized by all $\varphi$ 's approximants, the worlds are maximal consistent sets of formulas, and each set consists of the formulas that are true at it. For a given $S$, the partition classes consist of the worlds sharing the same formulas from $S$. Any two distinct worlds lie in distinct partition classes for all large enough $S$. So in the limit, as $S$ grows, the approximation becomes exact. Since all the approximants are valid in the canonical 
model, this means that its frame validates $\varphi$. Proving this is the chief technical contribution of the paper. The proof uses the special form of quasi-positive sentences and is similar to standard proofs of Sahlqvist's theorem.

Clearly, the same goes for the logic $\Lambda_{\Phi}$ axiomatized by the approximants to all the sentences in $\Phi$. Thus, $\Lambda_{\Phi}$ is sound and complete for the class of frames in which $\Phi$ is valid. This class is elementary, so $\Lambda_{\Phi}$ is elementarily generated. It contains the canonical frame for $\Lambda_{\Phi}$, so $\Lambda_{\Phi}$ is canonical. Since every elementarily generated logic is of the form $\Lambda_{\Phi}$, this provides another proof of Fine's theorem [7].

1.5 How natural are the axioms? Methods of synthesizing axioms from a template are often accused of producing artificial, unenlightening axiomatizations. What about our method? Well, the set $U$ of axioms obtained in step 1 can be extremely opaque, and it is infinite even if the original $T$ is finite. The sentences in $U$ are similar in structure to pure quasi-positive sentences and the translation between them in step 2 is fairly straightforward, though it can increase the opacity a little. But sometimes we can start off with a class of frames defined by pure quasi-positive hybrid sentences. Steps 1 and 2 are omitted, and the modal axioms are synthesized directly from the hybrid ones. We actually get to see the axioms, and they can be rather natural and easily understood. Consider, for example, the logics $K M^{\infty}$ and $K M T$ discussed above. The quasi-positive sentence $\diamond \exists i \square i$ expresses (1), and $\diamond \exists i(i \wedge \diamond i)$ expresses (3), and the approximants obtained from them are equivalent to the known axioms (2), (4), respectively. For instance, the axiom obtained by approximating $\diamond \exists i \square i$ with respect to $S=\left\{p_{1}, \ldots, p_{n}\right\}$ is

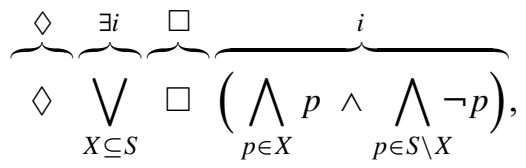

and this is equivalent to the $M_{n}$ of (2). See Examples 4.6 and 4.7 for more details. If we can obtain, modulo simple equivalences, axiomatizations that are already known from the literature, then it seems fair to say that sometimes our method produces fairly natural and intuitive axiomatizations.

This allows us to make a small contribution to problem (A) above. Given some known axioms for a modal logic, if we can devise an elementary class whose modal axioms (obtained by our method) are equivalent to the given ones, we have at hand a proof that the logic is elementarily generated, and by a specific elementary class of frames. This is not quite an idle dream: the method presented here originated in study of the examples $K M^{\infty}$ and $K M T$.

1.6 Comparison with Sahlqvist's completeness theorem Sahlqvist's completeness theorem ([32], [33], [3]) says that the logic axiomatized by a Sahlqvist formula $\sigma$ is the logic of the class of frames in which $\sigma$ is valid. Step 3 above says that the logic $\Lambda_{\varphi}$ axiomatized by the approximants of a pure quasi-positive sentence $\varphi$ is the logic of the class of frames that validate $\varphi$. Both results generalize to sets of formulas.

We view step 3 as extending Sahlqvist's completeness theorem. First, every Sahlqvist formula can be expressed by (is valid in the same frames as) a quasipositive one. Second, the proof that step 3 works (Proposition 5.9 below) parallels known proofs of Sahlqvist's theorem rather closely. We do not need to use negated 
boxed atoms and the "minimal assignments" associated with them, so the most direct analogy is perhaps with [26], which proves that positive equations are preserved by algebraic canonical extensions. But with some extra work we could allow $\varphi$ to be a "hybrid Sahlqvist formula." Step 3 would then simply extend Sahlqvist's completeness theorem to this larger class of formulas. In the special case where $\varphi$ is a modal Sahlqvist formula, the approximants would be simply the substitution instances of $\varphi$. We will discuss this in Example 3.6, Remark 3.16, and Section 6.2. It is not necessary to put in this extra work because it does not increase the range of logics we can axiomatize, though it would simplify some of the axiomatizations.

There are, however, some differences between our result and Sahlqvist's. For one, it generates modal axioms and a modal logic $\Lambda_{\varphi}$ from a hybrid formula $\varphi$. This provides a perhaps new and surprising connection between modal and hybrid logic. Because $\varphi$ generates purely modal axioms, our result is not to be regarded as a Sahlqvist theorem for hybrid logic; readers seeking such a theorem should consult, for example, [18] and [34].

It is also perhaps notable that the set of axioms for $\Lambda_{\varphi}$ is infinite. In a way, this is not so alarming. First, the logics $K M^{\infty}, K M T$, and so on, are not finitely axiomatizable, so infinite axiomatizations are inevitable if we are going to cover them. One benefit of allowing infinite axiomatizations is that we can after all "explain" the canonicity of these logics by Sahlqvist-like reasons, even though they have no axiomatization by canonical formulas. Second, the infiniteness is to an extent already present in the standard Sahlqvist theorem. For example, the logic $K 4$ generated by the Sahlqvist axiom $\square p \rightarrow \square \square p$ is in a way axiomatized by the infinite set of substitution instances $\{\square \alpha \rightarrow \square \square \alpha: \alpha$ a modal formula\}. $K 4$ is finitely axiomatizable only because of the presence of the substitution rule. Alternatively, $\square p \rightarrow \square \square p$ can be regarded as a schema, from which axioms $\square \alpha \rightarrow \square \square \alpha$ are generated. But a quasi-positive sentence is also a schema in the sense that it generates an infinite number of modal axioms in a purely syntactic and effective way. The difference from conventional schemata is only that the generating process is a little more complicated than substitution.

Nonetheless, some logics $\Lambda_{\varphi}$ are finitely axiomatizable, whereas we only obtain an infinite set of axioms for them. How to determine whether $\Lambda_{\varphi}$ is finitely axiomatizable and how to obtain a finite set of axioms when it is remain interesting questions for the future.

1.7 Comparison with Sahlqvist's correspondence theorem This part of Sahlqvist's theorem says that for any Sahlqvist formula $\sigma$ there is a first-order sentence, the "correspondent" of $\sigma$, that is true in precisely the frames in which $\sigma$ is valid. The correspondent is obtained from $\sigma$ by a simple algorithm. To compare this with our results, we need to distinguish between the class Fr $\Phi$ of frames validating $\Phi$ (the class $\overline{\mathcal{K}}$ of step 2), and the class $\operatorname{Fr} \Lambda_{\Phi}$ of frames validating $\Lambda_{\Phi}$. Their analogues for a Sahlqvist formula $\sigma$ are the class of models of the correspondent of $\sigma$ and the class of frames for $\sigma$, respectively, and Sahlqvist's theorem gave us the bonus that these two classes are the same. In our situation, we only have $\operatorname{Fr} \Phi \subseteq \operatorname{Fr} \Lambda_{\Phi}$, and the inclusion can be strict. For example, there are frames validating $K M^{\infty}$ and $K M T$ that do not satisfy the conditions (1) and (3), respectively. (For which $\Phi$ is the inclusion strict is another question for the future.) 
For the class $\operatorname{Fr} \Phi$, correspondence is trivial, because quasi-positive sentences have straightforward first-order translations: they are essentially their own first-order correspondents. For Fr $\Lambda_{\Phi}$, no first-order correspondence is possible in general, because $\operatorname{Fr} \Lambda_{\Phi}$ can be nonelementary. This is so for $K M^{\infty}$ ([15], [2]) and, in an algebraic setting, for RRA [22]. The class of nonfinitely colorable graphs is nonelementary [22], and this can be used to show that Fr KMT is nonelementary as well.

So we cannot think of $\Phi$ as a set of correspondents of $\Lambda_{\Phi}$ : half our "Sahlqvist bonus" can be forfeited. But the other half we get to keep: $\Phi$ is valid in the canonical frame for $\Lambda_{\Phi}$. Since $\Lambda_{\Phi}$ is valid in any frame validating $\Phi$, the two are canonical pseudo-correspondents in the sense of [39].

1.8 Summary We give a necessary and sufficient syntactic condition for a modal logic to be elementarily generated and one that matches existing axiomatizations of some known examples quite closely. However, it yields infinite axiomatizations of even finitely axiomatizable modal logics. Nonetheless, we hope it will be useful in showing more modal logics to be canonical and proving completeness theorems for them.

1.9 Layout of paper The three steps in Subsection 1.4 are treated in the order 2, 3, 1. Step 1 can be found in Theorem 5.16, step 2 in Theorem 3.13, and step 3 in Theorem 5.15. In Section 2, we set out the definitions of modal and hybrid formulas. In Section 3, we introduce the quasi-positive formulas that will be our main concern. We give some examples and prove that every positive relativized first-order sentence can be expressed by such a formula. Section 4 defines the approximants of a hybrid formula, gives some examples, and proves some basic facts about them. The main Sahlqvist-like proof of soundness and completeness of the logic axiomatized by the approximants of quasi-positive formulas is in Section 5; Subsection 5.5 shows that every elementarily generated logic can be handled in this way. Section 6 has a discussion of the results and some open questions.

\section{Modal and Hybrid Formulas}

Our paper is fairly self-contained, but we do envisage that the reader has some prior acquaintance with modal and hybrid logic. Background information can be found in, for example, [3].

We consider any modal language $\mathcal{L}$ over the fixed infinite set $\mathcal{V}$ of propositional atoms (or propositional variables). For simplicity, we assume that $\mathcal{L}$ has a single $k$-ary box $\square$ and a corresponding diamond $\diamond$, where $k \geq 1$. All our proofs extend to languages $\mathcal{L}$ with multiple modalities, and occasionally we will add extra modalities to $\mathcal{L}$.

$\mathcal{L}^{+}$denotes the extension of $\mathcal{L}$ with nominals and the nominal quantifiers. Formally, we fix a set $\ell$ of nominals disjoint from $\mathcal{V}$. (More properly, they should be called state variables.)

Definition 2.1 The $\mathscr{L}^{+}$-formulas (over $\mathcal{V} \cup \ell$ ) are defined as follows. The atomic $\mathcal{L}^{+}$-formulas are $q$, for any $q \in \mathcal{V} ; i$, for any $i \in \mathcal{l}$; and $\mathrm{\top}, \perp$. If $\varphi, \psi, \varphi_{1}, \ldots, \varphi_{k}$ are $\mathcal{L}^{+}$-formulas, then so are $\neg \varphi, \varphi \wedge \psi, \varphi \vee \psi, \square\left(\varphi_{1}, \ldots, \varphi_{k}\right), \diamond\left(\varphi_{1}, \ldots, \varphi_{k}\right)$, and also $\forall i \varphi$ and $\exists i \varphi$, for any nominal $i . \mathscr{L}$ is the fragment of $\mathcal{L}^{+}$without nominals or quantifiers. 
An occurrence of a nominal $i$ in a formula $\varphi$ is said to be bound if it in the scope of a quantifier $\forall i$ or $\exists i$ in $\varphi$, and free, otherwise. For an $\mathcal{L}^{+}$-formula $\varphi$, we write free $(\varphi)$ for the set of all atoms $a \in \mathcal{V}$ that occur in $\varphi$ and all nominals $i \in \mathcal{l}$ that have free occurrences in $\varphi$. An $\mathscr{L}^{+}$-formula is a sentence if it has no free occurrences of nominals and pure if it has no occurrences of atoms.

As usual, $\varphi \rightarrow \psi$ abbreviates $\neg \varphi \vee \psi$, and $\varphi \leftrightarrow \psi$ abbreviates $(\varphi \rightarrow \psi) \wedge(\psi \rightarrow \varphi)$. $\mathscr{L}$ and $\mathcal{L}^{+}$have the usual semantics. Formally, the definition for $\mathcal{L}^{+}$is as follows.

\section{Definition 2.2}

1. A frame is a structure of the form $\mathcal{F}=(W, R)$, where $R \subseteq W^{k+1}$. We will write $R\left(t, u_{1}, \ldots, u_{k}\right)$ to indicate that $\left(t, u_{1}, \ldots, u_{k}\right) \in R$.

2. For a frame $\mathcal{F}=(W, R)$ as above, a hybrid assignment into $\mathcal{F}$ is a map $h: \mathcal{V} \cup \ell \rightarrow \wp(W)$ such that $|h(i)|=1$ for each $i \in \ell$.

3. Given a hybrid assignment $h$ into $\mathcal{F}$ as above and a world $w \in W$, we define $\mathcal{F}, h, w \models \varphi$ by induction on $\mathcal{L}^{+}$-formulas $\varphi$ as follows.

(a) for $\varphi \in \mathcal{V} \cup \ell$, we put $\mathcal{F}, h, w \models \varphi$ iff $w \in h(\varphi)$.

(b) $T, \perp$, and Booleans - as usual.

(c) $\mathcal{F}, h, w \models \square\left(\varphi_{1}, \ldots, \varphi_{k}\right)$ iff for all $v_{1}, \ldots, v_{k} \in W$ with $R\left(w, v_{1}, \ldots, v_{k}\right)$, we have $\mathcal{F}, h, v_{l} \models \varphi_{l}$ for some $l$ with $1 \leq l \leq k$.

(d) $\mathcal{F}, h, w \models \diamond\left(\varphi_{1}, \ldots, \varphi_{k}\right)$ iff there are $v_{1}, \ldots, v_{k} \in W$ with $R\left(w, v_{1}, \ldots, v_{k}\right)$ and $\mathcal{F}, h, v_{l} \models \varphi_{l}$ for every $l$ with $1 \leq l \leq k$.

(e) $\mathcal{F}, h, w \models \forall i \varphi$ iff $\mathcal{F}, g, w \models \varphi$ for every hybrid assignment $g$ into $\mathcal{F}$ with $g(x)=h(x)$ for all $x \in \mathcal{V} \cup \ell \backslash\{i\}$.

(f) $\mathcal{F}, h, w \models \exists i \varphi$ iff $\mathcal{F}, g, w \models \varphi$ for some hybrid assignment $g$ into $\mathcal{F}$ with $g(x)=h(x)$ for all $x \in \mathcal{V} \cup \ell \backslash\{i\}$.

The definition of the semantics of the modal sublanguage $\mathcal{L}$ is an obvious variation on this; there, we only need a map $m: \mathcal{V} \rightarrow \wp(W)$, and we call such a map simply an assignment, or sometimes a modal assignment.

A formula $\varphi$ is said to be valid in a frame $\mathcal{F}$ if and only if $\mathcal{F}, h, w \models \varphi$ for all hybrid (or modal, as applicable) assignments $h$ into $\mathcal{F}$ and all worlds $w$ of $\mathcal{F}$.

Remark 2.3 In our view, $\forall i$ and $\exists i$ are quantifiers, not modalities. So $\mathcal{L}^{+}$has the same modal operators as $\mathcal{L}$. We have not included in $\mathcal{L}^{+}$the familiar hybrid binder $\downarrow$ or the actuality operator $@_{i}$. Recall their definitions:

1. $\mathcal{F}, h, w \models \downarrow_{i} \varphi$ iff $\mathcal{F}, g, w \models \varphi$, where $g$ is the same as $h$ except that $g(i)=\{w\}$

2. $\mathcal{F}, h, w \models @_{i} \varphi$ iff $\mathcal{F}, h, v \models \varphi$, where $h(i)=\{v\}$.

The first is no problem, since $\downarrow_{i} \varphi$ can be expressed in $\mathcal{L}^{+}$by $\exists i(i \wedge \varphi)$. The @ operator is more problematic. In the absence of quantifiers, it can be thought of as a box or diamond with accessibility relation $W \times\{v\}$. When the quantifiers $\forall i, \exists i$ are present, this relation can change, so then $@_{i}$ is not a conventional modality. However, in the case where $\mathcal{L}$ has universal modalities $A, E$ (i.e., a box and diamond with accessibility relation $W \times W)$, one can use either $A(i \rightarrow \varphi)$ or $E(i \wedge \varphi)$ to simulate $@_{i} \varphi$. Conversely, $E \varphi$ is definable from @ and $\exists$, via $\exists i @_{i} \varphi$, where $i$ does not occur free in $\varphi$, and $A \varphi$ is of course definable by $\neg E \neg \varphi$.

So one approach to @ is to use universal modalities to handle it. But it turns out that in many cases we can define @ in $\mathscr{L}^{+}$even without universal modalities. We will discuss this in Remark 3.16 below. 


\section{Quasi-positive Formulas}

These are roughly the positive hybrid formulas in which all universal quantification is relativized. The rest of the paper is largely devoted to studying them. In this section, we define them, give some examples, and study their expressive power.

\subsection{Definitions}

Notation 3.1 For $1 \leq d \leq k$, we let $\square^{d} \varphi$ abbreviate $\square(\perp, \ldots, \perp, \varphi, \perp, \ldots, \perp)$, and $\diamond^{d} \varphi$ abbreviate $\diamond(\top, \ldots, \top, \varphi, \top, \ldots, \top)$, in each case with the $\varphi$ in the $d$ th place.

Definition 3.2 The path formulas are defined as follows. Each path formula $\pi$ involves an atom, say $q$, and perhaps also nominals. We write it as $\pi(q)$ (of course, it may involve nominals as well as $q$ ). For a formula $\varphi$, we write $\pi(\varphi)$ for the result of replacing $q$ by $\varphi$ in $\pi(q)$.

1. Any atom $q$ is an existential path formula $\pi(q)$.

2. If $\pi(q)$ is an existential path formula, $1 \leq d \leq k$, and $j \in \ell$, then $\pi^{\prime}(q)=$ $\pi\left(\diamond^{d}(j \wedge q)\right)$ is an existential path formula.

3. If $\pi^{\diamond}(q)$ is an existential path formula, then the result $\pi^{\square}(q)$ of pushing negations down next to atomic formulas in $\neg \pi(\neg q)$ is a universal path formula, called the dual of $\pi^{\diamond}(q)$. For example, if $k=1$ and $\pi^{\diamond}(q)$ is $\diamond\left(j \wedge \diamond\left(j^{\prime} \wedge q\right)\right)$, then $\pi^{\square}(q)$ is $\square\left(j \rightarrow \square\left(j^{\prime} \rightarrow q\right)\right)$.

A typical existential path formula is $\pi \diamond(q)=\diamond^{d_{1}}\left(j_{1} \wedge \diamond^{d_{2}}\left(j_{2} \wedge \cdots \wedge \diamond^{d_{n}}\left(j_{n} \wedge q\right)\right) \cdots\right)$. It is rather like an @-operator. It is not hard to see that if $\mathcal{F}, h, w \models \pi^{\diamond}(i)$, where $i$ is a nominal with $h(i)=\{t\}$, say, then $\mathcal{F}, h, t \models \varphi$ if and only if $\mathcal{F}, h, w \models \pi^{\diamond}(\varphi)$, for any hybrid formula $\varphi$. So $\pi \diamond(\varphi)$ is like $@_{i} \varphi$. We will use this in Theorem 3.13.

Definition 3.3 The quasi-positive $\left(\mathcal{L}^{+}-\right)$formulas are defined as follows:

1. Any atomic $\mathcal{L}^{+}$-formula is quasi-positive.

2. If $\varphi, \psi$ are quasi-positive formulas, then so are $\varphi \wedge \psi$ and $\varphi \vee \psi$.

3. If $\varphi_{1}, \ldots, \varphi_{k}$ are quasi-positive, then so are $\square\left(\varphi_{1}, \ldots, \varphi_{k}\right)$ and $\diamond\left(\varphi_{1}, \ldots, \varphi_{k}\right)$.

4. If $\varphi$ is quasi-positive and $i \in \ell$, then $\exists i \varphi$ is quasi-positive.

5. If $\varphi$ is a quasi-positive formula, $\pi \diamond(q)$ is an existential path formula and $\pi^{\square}(q)$ the dual universal path formula, and $i_{1}, \ldots, i_{k} \in \ell \backslash$ free $\left(\pi^{\diamond}(q)\right)$, then

$$
\pi^{\diamond}(\top) \wedge \forall i_{1} \ldots i_{k}\left(\pi^{\square}\left(\diamond\left(i_{1}, \ldots, i_{k}\right)\right) \rightarrow \varphi\right)
$$

is also a quasi-positive formula.

It will become clear in Proposition 5.9 why we use $\pi^{\square}$ in this way in the last clause, even though semantically it would be equivalent to use $\pi^{\diamond}$. Unfortunately, we have not been able to find a simpler formulation of universal quantifiers that allows Proposition 5.9 to be proved. Remark 5.11 has some discussion of this.

We could also allow formulas like $\forall i(i \rightarrow \varphi)$, but this is equivalent to $\exists i(i \wedge \varphi)$, so there is no need to.

3.2 Examples We will see that quasi-positive formulas are rather expressive. We give some examples; for simplicity, we assume here that the arity $k$ of $\square$ and $\diamond$ is 1 . 
Example 3.4 $\diamond \exists i \square i$ says that there is a successor that sees at most one world. It is a quasi-positive sentence. Similarly, $\diamond \exists i \exists j \square(i \vee j)$ says that there is a successor with at most two successors.

Example 3.5 $\diamond \exists i(i \wedge \triangleright i)$ says that there is a reflexive successor. Similarly, if $\mathcal{L}$ has universal modalities $A, E$, the quasi-positive sentences $E \exists i(i \wedge \triangleright i)$ and $A \exists i(i \wedge \triangleright i)$ say that some (respectively, all) worlds are reflexive.

Example 3.6 Modal Sahlqvist formulas such as $\square p \rightarrow p$ and $\diamond \square p \rightarrow \square \diamond p$ are valid in the same frames as

$$
\exists i(i \wedge \diamond i) \text { and } \forall i\left(\diamond i \rightarrow \forall i^{\prime}\left[\diamond i^{\prime} \rightarrow \exists j\left(\diamond(i \wedge \diamond j) \wedge \diamond\left(i^{\prime} \wedge \diamond j\right)\right)\right]\right) .
$$

There seems to be no absolutely standard definition of a modal Sahlqvist formula, but probably a reasonably well-accepted definition is the one in the recent textbook [3]. In slightly restricted and simplified form, it says that

1. any positive modal formula is a Sahlqvist formula,

2. any negated boxed atom $\neg \square^{n} p$ (where $\square^{0} p=p$ and $\square^{n+1} p=\square\left(\square^{n} p\right)$ ) is a Sahlqvist formula,

3. if $\varphi, \psi$ are Sahlqvist formulas then so are $\varphi \wedge \psi, \varphi \vee \psi$, and $\square \varphi$.

For every Sahlqvist formula, there is a pure quasi-positive sentence that is valid in the same frames: see Remark 3.16 below. As mentioned in Section 1, we can even generalize the definition of Sahlqvist formula in a natural way to hybrid logic by allowing quasi-positive hybrid sentences instead of positive modal formulas in (1). Even then, every such formula can be rewritten as a pure quasi-positive sentence that is valid in the same frames. We will not prove this here, but see Section 6.2 for a little more discussion of this point.

Example 3.7 In fact, quasi-positive formulas go beyond Sahlqvist ones in expressiveness. As we said in the Introduction, [3], p. 168, shows that the axiom ' $M 4$ ' $=(\square \diamond p \rightarrow \diamond \square p) \wedge(\square p \rightarrow \square \square p)$ is not equivalent to any Sahlqvist formula. However, the authors show that it is valid in precisely the transitive frames satisfying

$$
(*) \quad \forall x \exists y(R(x, y) \wedge \forall z t(R(y, z) \wedge R(y, t) \rightarrow z=t)) .
$$

And so is the pure quasi-positive sentence

$$
\underbrace{\forall j(\diamond j \rightarrow(\diamond j \wedge \forall i(\square(j \rightarrow \diamond i) \rightarrow \diamond i)))}_{\text {transitivity }} \wedge \underbrace{\diamond \exists i \square i}_{(*)} .
$$

The hybrid rendition of $(*)$ is borrowed from Example 3.4 above.

Example 3.8 The Sahlqvist formula $\diamond \square p \rightarrow \diamond p$ has first-order correspondent

$$
\forall y z(R(y, z) \rightarrow \exists t(R(z, t) \wedge R(y, t))) .
$$

The similar statement

$$
\forall x \exists y(R(x, y) \wedge \forall z(R(y, z) \rightarrow \exists t(R(z, t) \wedge R(y, t))))
$$

does not appear to be Sahlqvist-expressible $(\diamond(\diamond \square p \rightarrow \diamond p)$ is not Sahlqvist); but we can express it in hybrid logic by

$$
\diamond \exists i\left(i \wedge \square \diamond \exists j\left(j \wedge @_{i} \diamond j\right)\right),
$$


and also by the quasi-positive formula

$$
\diamond \forall j(\diamond j \rightarrow \exists k(\diamond(j \wedge \diamond k) \wedge \diamond k)) .
$$

Notice how the $\square$ in the first formula is simulated by $\forall j$ in the second.

Example 3.9 Irreflexivity: neither $\exists i(i \wedge \square \neg i)$ nor the equivalent $\forall i(i \rightarrow \square \neg i)$ are equivalent to pure quasi-positive sentences. See Section 6.4 for a proof.

Example 3.10 $\forall i$ i is not quasi-positive, because the $\forall i$ is not relativized. We will see in Remark 5.11 that it lacks some useful properties of quasi-positive formulas and is not equivalent to a quasi-positive formula.

3.3 Expressiveness of quasi-positive formulas We now analyze the expressiveness of quasi-positive formulas in more detail. We begin with a definition from [12].

Definition 3.11 Let $L$ be the first-order signature consisting of a $(k+1)$-ary relation symbol $R$. An essentially atomic $L$-formula $\psi$ is one built from atomic $L$-formulas (which are of the form $R\left(x, y_{1}, \ldots, y_{k}\right), x=y, \top$, and $\perp$ ) using $\wedge, \vee$, and bounded quantification

$$
\begin{aligned}
& \forall y_{1}, \ldots, y_{k}\left(R\left(x, y_{1}, \ldots, y_{k}\right) \rightarrow \psi\right), \\
& \exists y_{1}, \ldots, y_{k}\left(R\left(x, y_{1}, \ldots, y_{k}\right) \wedge \psi\right),
\end{aligned}
$$

where $x \notin\left\{y_{1}, \ldots, y_{k}\right\}$. An $L$-sentence is pseudo-equational if it is of the form $\forall x \psi$, where $\psi$ is essentially atomic.

We will regard a frame $(W, R)$ (Definition 2.2) as an $L$-structure in the natural way. So we can evaluate essentially atomic formulas and pseudo-equations in frames. ${ }^{4}$

Remark 3.12 Given any pure quasi-positive hybrid sentence $\varphi$, it is not hard to construct a pseudo-equational $L$-sentence that is true in precisely the frames in which $\varphi$ is valid. A quasi-positive formula may have unrelativized existential quantification of the form $\exists i \varphi$, but because $\varphi$ is essentially a positive formula, this is only of use if the value taken up by $i$ is "reachable" from the evaluation point of $\varphi$ by a number of "steps" at most the modal depth of $\varphi$. So we may simulate $\exists i$ by a disjunction of relativized existential quantifiers sufficient to reach all points at most this far away. For example, $\exists i \square \diamond i$ is valid in the same frames as the pseudo-equational sentence

$$
\begin{aligned}
\forall x(\forall y(R(x, y) \rightarrow & R(y, x)) \\
& \vee \exists i[R(x, i) \wedge \forall y(R(x, y) \rightarrow R(y, i))] \\
& \vee \exists j(R(x, j) \wedge \exists i[R(j, i) \wedge \forall y(R(x, y) \rightarrow R(y, i))])) .
\end{aligned}
$$

We leave the general argument as an exercise, since we will not use it.

More importantly here, the converse also holds, as we now show. The very simple idea seen in Examples 3.7 and 3.8 is at the root of this theorem.

Theorem 3.13 For any pseudo-equational L-sentence $\sigma$, there is an effectively constructible pure quasi-positive hybrid $\mathcal{L}^{+}$-sentence $\varphi$ such that for any frame $\mathcal{F}$, we have $\mathcal{F} \models \sigma$ if and only if $\varphi$ is valid in $\mathcal{F}$.

Proof In the proof, we make no notational distinction between first-order variables and nominals. If $\mathcal{F}=(W, R)$ is a frame, $h$ is a map from the first-order variables (identified with nominals) into $W$, and $\varphi$ is a pure hybrid formula, we write 
$\mathcal{F}, h, w \models_{H} \varphi$ if $\mathcal{F}, \widehat{h}, w \models \varphi$, where $\widehat{h}$ is the hybrid assignment into $\mathcal{F}$ given by $\widehat{h}(i)=\{h(i)\}$ for $i \in \ell$ (and $\widehat{h}(p)=\varnothing$ for $p \in \mathcal{V})$.

Fix a distinguished variable (nominal) $x$. Let $\psi$ be a first-order $L$-formula. We write free $\psi$ for the set of free variables of $\psi$. If $\alpha=\left\langle\pi_{v}(q): v \in V\right\rangle$ is a sequence of existential path formulas indexed by a set $V$ of variables, we say that $\psi$ is $\alpha$-safe if

1. $V \supseteq$ free $\psi$,

2. for each subformula $\psi^{\prime}$ of $\psi$, no variable has both free and bound occurrences in $\psi^{\prime}$,

3. no variable in $V \cup\{x\}$, nor any nominal in any $\pi_{v}^{\diamond}(\top)$ for any $v \in$ free $\psi$, occurs bound in $\psi$.

Claim 3.14 Let $\psi$ be an essentially atomic $L$-formula. Let $V$ be a set of variables and let $\alpha=\left\langle\pi_{v}^{\diamond}(q): v \in V\right\rangle$ be a sequence of existential path formulas. If $\psi$ is $\alpha$-safe, then we can effectively construct a quasi-positive hybrid formula $\psi^{\alpha}$ such that for any frame $\mathcal{F}$ and any $h$ with $\mathcal{F}, h, h(x) \models_{H} \pi_{v}^{\diamond}(v)$ for each $v \in$ free $\psi$, we have

$$
\mathcal{F}, h \models \psi \Longleftrightarrow \mathcal{F}, h, h(x) \models_{H} \psi^{\alpha} .
$$

Proof of claim We define $\psi^{\alpha}$ by induction on $\psi$. In Example 3.15 below, we will give an example of the construction. For atomic $\psi$, there are three cases:

1. $R\left(y, z_{1}, \ldots, z_{k}\right)^{\alpha}=\pi_{y}^{\diamond}\left(\diamond\left(z_{1}, \ldots, z_{k}\right)\right)$,

2. $(y=z)^{\alpha}=\pi_{y}^{\diamond}(z)$,

3. $\top^{\alpha}=\top$ and $\perp^{\alpha}=\perp$.

Let $\mathcal{F}, h$ be arbitrary. It is easily seen that for any variable $y$ and any hybrid formula $\varphi$, if $\mathcal{F}, h, h(x) \models_{H} \pi_{y}^{\diamond}(y)$ then

$$
\mathcal{F}, h, h(y) \models_{H} \varphi \Longleftrightarrow \mathcal{F}, h, h(x) \models_{H} \pi_{y}^{\diamond}(\varphi) .
$$

So assuming that $\mathcal{F}, h, h(x) \models_{H} \pi_{y}^{\diamond}(y)$, and writing $\bar{z}$ for $\left(z_{1}, \ldots, z_{k}\right)$, we evidently have

1. $\mathcal{F}, h \models R(y, \bar{z})$ iff $\mathcal{F}, h, h(y) \models_{H} \diamond \bar{z}$, iff $\mathcal{F}, h, h(x) \models_{H} \pi_{y}^{\diamond}(\diamond \bar{z})$,

2. $\mathcal{F}, h \models y=z$ iff $\mathcal{F}, h, h(y) \models_{H} z$, iff $\mathcal{F}, h, h(x) \models_{H} \pi_{y}^{\diamond}(z)$.

Therefore, (7) holds for atomic $\psi$.

The translation $-{ }^{\alpha}$ commutes with $\wedge$ and $\vee$. If $\psi_{1} \wedge \psi_{2}$ is $\alpha$-safe, so are $\psi_{1}$ and $\psi_{2}$. Inductively, $\psi_{1}^{\alpha}$ and $\psi_{2}^{\alpha}$ are well defined and satisfy (7). If $\mathcal{F}, h, h(x) \models_{H} \pi_{v}^{\diamond}(v)$ for each $v \in$ free $\left(\psi_{1} \wedge \psi_{2}\right)$, then the same holds for $\psi_{1}$ and $\psi_{2}$ individually; so $\mathcal{F}, h \models \psi_{1} \wedge \psi_{2}$ if and only if $\mathcal{F}, h, h(x) \models_{H} \psi_{1}^{\alpha} \wedge \psi_{2}^{\alpha}$, as required. The case of $\vee$ is similar.

Finally, let $\psi$ be essentially atomic, and suppose that the quasi-positive formula $\psi^{\alpha}$ has been defined for all $\alpha$ for which $\psi$ is safe. Suppose that $\alpha=\left\langle\pi_{v}^{\diamond}: v \in V\right\rangle$ is given. We extend the definition to quantifiers as follows:

1. Let $\theta=\exists z_{1}, \ldots, z_{k}\left(R\left(y, z_{1}, \ldots, z_{k}\right) \wedge \psi\right)$, and suppose that $\theta$ is $\alpha$-safe. Then

$$
\theta^{\alpha}=\exists z_{1} \ldots z_{k}\left(\pi_{y}^{\diamond}\left(\diamond\left(z_{1}, \ldots, z_{k}\right)\right) \wedge \psi^{\beta}\right) .
$$

2. Let $\theta=\forall z_{1}, \ldots, z_{k}\left(R\left(y, z_{1}, \ldots, z_{k}\right) \rightarrow \psi\right)$, with $\theta \alpha$-safe. Then

$$
\theta^{\alpha}=\pi_{y}^{\diamond}(\top) \wedge \forall z_{1} \ldots z_{k}\left(\pi_{y}^{\square}\left(\diamond\left(z_{1}, \ldots, z_{k}\right)\right) \rightarrow \psi^{\beta}\right) .
$$


In each case, $y \notin\left\{z_{1}, \ldots, z_{l}\right\}$, and the sequence $\beta$ is the extension of $\alpha$ with the path formulas $\pi_{z_{1}}^{\diamond}(q), \ldots, \pi_{z_{k}}^{\diamond}(q)$, with

$$
\pi_{z l}^{\diamond}(q)=\pi_{y}^{\diamond}\left(\diamond^{l}\left(z_{l} \wedge q\right)\right) \quad \text { for } l=1, \ldots, k
$$

As $\theta$ is $\alpha$-safe and $z_{l}$ occurs bound in $\theta$, there is no path formula indexed by $z_{l}$ already in $\alpha$. So $\beta$ is indeed indexed by a set of variables. It can be checked that $\psi$ is $\beta$-safe. Inductively, $\psi^{\beta}$ is a well-defined quasi-positive formula, and so in each case, $\theta^{\alpha}$ is also well defined and quasi-positive.

We will check that (7) is preserved by these two definitions. We need some preliminaries. The set free $(\theta)$ is the same in each case. Let $\mathcal{F}, h$ be given, with $\mathcal{F}, h, h(x) \models_{H} \pi_{v}^{\diamond}(v)$ for each $v \in$ free $(\theta)$. Write $\bar{z}=\left(z_{1}, \ldots, z_{k}\right)$, and write $g=\bar{z} h$ if $g$ is a map from variables into $W$ that agrees with $h$ except perhaps on $\bar{z}$. For any such $g$, we have the following.

(a) $g(x)=h(x)-\theta$ is $\alpha$-safe, so $x$ does not occur bound in $\theta$, so $x \notin\left\{z_{1}, \ldots, z_{k}\right\}$.

(b) $\mathcal{F}, g, g(x) \models_{H} \pi_{v}^{\diamond}(v)$ for each $v \in$ free $\theta$. To see this, let $v \in$ free $\theta$. As $\theta$ is assumed $\alpha$-safe, neither $v$ nor the nominals in $\pi_{v}^{\diamond}(T)$ are in $\left\{z_{1}, \ldots, z_{k}\right\}$, and so $g$ and $h$ agree on them. We assumed that $\mathcal{F}, h, h(x) \models_{H} \pi_{v}^{\diamond}(v)$, so by (a), we must also have $\mathcal{F}, g, g(x) \models_{H} \pi_{v}^{\diamond}(v)$.

(c) $\mathcal{F}, g \models R(y, \bar{z})$ iff $\mathcal{F}, g, g(x) \models_{H} \pi_{y} \diamond(\diamond \bar{z})$. Certainly, $\mathcal{F}, g \models R(y, \bar{z})$ iff $\mathcal{F}, g, g(y) \models_{H} \diamond \bar{z}$. Since $y \in$ free $\theta$, by (b) we have $\mathcal{F}, g, g(x) \models_{H} \pi_{y}^{\diamond}(y)$. So it is clear by (8) that the above holds iff $\mathcal{F}, g, g(x) \models_{H} \pi_{y}^{\diamond}(\diamond \bar{z})$.

(d) If $\mathcal{F}, g \models R(y, \bar{z})$, then $\mathcal{F}, g \models \psi$ iff $\mathcal{F}, g, g(x) \models_{H} \psi^{\beta}$. It is clear that the latter condition in (c) implies $\mathcal{F}, g, g(x) \models_{H} \pi_{z_{l}}^{\diamond}\left(z_{l}\right)$ for $l=1, \ldots, k$. By (b) and free $\psi \subseteq\left\{z_{1}, \ldots, z_{k}\right\} \cup$ free $\theta$, we see that $\mathcal{F}, g, g(x) \models_{H} \pi_{v}^{\diamond}(v)$ for all $v \in$ free $\psi$. The conclusion now follows inductively from (7).

We can now check (7) for the new formulas. First, let $\theta=\exists \bar{z}(R(y, \bar{z}) \wedge \psi)$. Then $\mathcal{F}, h \models \theta$ if and only if there is $g=\bar{z} h$ such that $\mathcal{F}, g \models R(y, \bar{z}) \wedge \psi$. By (c) and (d), this holds if and only if there is $g=\bar{z} h$ with $\mathcal{F}, g, g(x) \models_{H} \pi_{y}^{\diamond}(\diamond \bar{z}) \wedge \psi^{\beta}$. Clearly, this is if and only if $\mathcal{F}, h, h(x) \models_{H} \theta^{\alpha}$.

Second, let $\theta=\forall \bar{z}(R(y, \bar{z}) \rightarrow \psi)$. Let $g=\bar{z} h$. By (c), $\mathcal{F}, g \models R(y, \bar{z})$ if and only if $\mathcal{F}, g, g(x) \models_{H} \pi_{y}^{\diamond}(\diamond \bar{z})$. By (b), $\mathcal{F}, g, g(x) \models_{H} \pi_{y}^{\diamond}(y)$, so this holds if and only if $\mathcal{F}, g, g(x) \models_{H} \pi_{y}^{\square}(\diamond \bar{z})$. By this and (d), we see that $\mathcal{F}, g \models R(y, \bar{z}) \rightarrow \psi$ is equivalent to $\mathcal{F}, g, g(x) \models_{H} \pi_{y}^{\square}(\diamond \bar{z}) \rightarrow \psi^{\beta}$.

It is now plain that $\mathcal{F}, h \models \theta$ if and only if $\mathcal{F}, h, h(x) \models_{H} \forall \bar{z}\left(\pi_{y}^{\square}(\diamond \bar{z}) \rightarrow \psi^{\beta}\right)$. It follows from our assumptions that $\mathcal{F}, h, h(x) \models_{H} \pi_{y}^{\diamond}(\top)$, so we obtain $\mathcal{F}, h \models \theta$ if and only if $\mathcal{F}, h, h(x) \models_{H} \theta^{\alpha}$, as required. This proves the claim.

Now suppose that we are given some essentially atomic formula $\psi(x)$. Define $\pi_{x}^{\diamond}(q)=q$. By renaming bound variables, we may equivalently rewrite $\psi$ so that it is $\left\langle\pi_{x}^{\diamond}\right\rangle$-safe. Let $\varphi$ be $\exists x\left(x \wedge \psi^{\left\langle\pi_{x}^{\diamond}\right\rangle}\right)$. It can be checked that for each $\psi$ as in the claim, each free nominal of $\psi^{\alpha}$ is a free variable of $\psi$ or occurs in $\pi_{y}^{\diamond}(\top)$ for some $y \in$ free $\psi$. Hence, $\varphi$ is a pure quasi-positive sentence. By definition, $\varphi$ is valid in a frame $\mathcal{F}=(W, R)$ if and only if $\mathcal{F}, h, w \models_{H} \varphi$ for every $h$ and $w \in W$, if and only if for every $h, w$ we have $\mathcal{F}, g, g(x) \models_{H} \psi^{\left\langle\pi_{x}^{\diamond}\right\rangle}$, where $g$ is the same as $h$ except that $g(x)=w$. This boils down to saying that $\mathcal{F}, g, g(x) \models_{H} \psi^{\left\langle\pi_{x}^{\diamond}\right\rangle}$ for every $g$. 
Now $\pi_{x}^{\diamond}(x)=x$, and hence $\mathcal{F}, g, g(x) \models_{H} \pi_{x}^{\diamond}(x)$. So by the claim, for any $g$ we have $\mathcal{F}, g, g(x) \models_{H} \psi^{\left\langle\pi_{x}^{\diamond}\right\rangle}$ if and only if $\mathcal{F}, g \models \psi$. We conclude that $\varphi$ is valid in $\mathcal{F}$ if and only if $\mathcal{F}, g \models \psi$ for every $g$-and this is if and only if $\mathcal{F} \models \forall x \psi(x)$. As $\mathcal{F}$ was arbitrary, the theorem is proved.

Example 3.15 The construction in the theorem may seem elaborate, but it is actually quite simple. We give an example to illustrate it. We assume here that $k=1$. The symbol $\equiv$ will denote logical equivalence. Consider the pseudo-equational sentence $\forall x \psi(x)$, where

$$
\psi(x)=\exists y(R(x, y) \wedge \forall z(R(y, z) \rightarrow R(x, z) \vee R(z, x) \vee x=z)) .
$$

We put $\pi_{x}^{\diamond}(q)=q$, and apply the algorithm of the claim in Theorem 3.13 to calculate $\psi^{\left\langle\pi_{x}^{\diamond}\right\rangle}$. Let $\psi_{1}=\forall z(R(y, z) \rightarrow R(x, z) \vee R(z, x) \vee x=z)$. As in the $\exists$-case in the claim, we put

$$
\begin{aligned}
\pi_{y}^{\diamond}(q) & =\pi_{x}^{\diamond}(\diamond(y \wedge q))=\diamond(y \wedge q), \\
\psi^{\left\langle\pi_{x}^{\diamond}\right\rangle} & =\exists y\left(\pi_{x}^{\diamond}(\diamond y) \wedge \psi_{1}^{\left\langle\pi_{x}^{\diamond}, \pi_{y}^{\diamond}\right\rangle}\right) \\
& =\exists y\left(\diamond y \wedge \psi_{1}^{\left\langle\pi_{x}^{\diamond}, \pi_{y}^{\diamond}\right\rangle}\right) .
\end{aligned}
$$

Then, with $\psi_{2}=R(x, z) \vee R(z, x) \vee x=z$, the claim dictates that we put

$$
\begin{aligned}
& \pi_{y}^{\square}(q)=\square(y \rightarrow q), \\
& \pi_{z}^{\diamond}(q)=\pi_{y}^{\diamond}(\diamond(z \wedge q))=\diamond(y \wedge \diamond(z \wedge q)), \\
& \psi_{1}^{\left\langle\pi_{x}^{\diamond}, \pi_{y}^{\diamond}\right\rangle}=\pi_{y}^{\diamond}(\top) \wedge \forall z\left(\pi_{y}^{\square}(\diamond z) \rightarrow \psi_{2}^{\left.\left\langle\pi_{x}^{\diamond}, \pi_{y}^{\diamond}, \pi_{z}^{\diamond}\right\rangle\right)}\right. \\
& \equiv \diamond y \wedge \forall z\left([\square(y \rightarrow \diamond z)] \rightarrow \psi_{2}^{\left\langle\pi_{x}^{\diamond}, \pi_{y}^{\diamond}, \pi_{z}^{\diamond}\right\rangle}\right) .
\end{aligned}
$$

Finally, we obtain

$$
\begin{aligned}
& \psi_{2}^{\left\langle\pi_{x}^{\diamond}, \pi_{y}^{\diamond}, \pi_{z}^{\diamond}\right\rangle}=(R(x, z) \vee R(z, x) \vee x=z)^{\left\langle\pi_{x}^{\diamond}, \pi_{y}^{\diamond}, \pi_{z}^{\diamond}\right\rangle} \\
& =\pi_{x}^{\diamond}(\diamond z) \vee \pi_{z}^{\diamond}(\diamond x) \vee \pi_{x}^{\diamond}(z) \\
& =\diamond z \vee \diamond(y \wedge \diamond(z \wedge \diamond x)) \vee z \text {. }
\end{aligned}
$$

Wrapping up and simplifying, we obtain

$$
\psi^{\left\langle\pi_{x}^{\diamond}\right\rangle} \equiv \exists y(\diamond y \wedge \forall z([\square(y \rightarrow \diamond z)] \rightarrow \diamond z \vee \diamond(y \wedge \diamond(z \wedge \diamond x)) \vee z)) .
$$

Recalling that

$$
\psi(x)=\exists y(R(x, y) \wedge \forall z(R(y, z) \rightarrow R(x, z) \vee R(z, x) \vee x=z)),
$$

it is not so hard to see that for any $\mathcal{F}, h$, we have $\mathcal{F}, h \models \psi$ if and only if $\mathcal{F}, h, h(x) \models_{H} \psi^{\left\langle\pi_{x}^{\diamond}\right\rangle}$, as in the claim, and that $\exists x\left(x \wedge \psi^{\left\langle\pi_{x}^{\diamond}\right\rangle}\right)$ is valid in $\mathcal{F}$ if and only if $\mathcal{F} \models \forall x \psi(x)$.

Remark 3.16 If we extend the formation rules in Definition 3.11 by allowing negation, we obtain what is sometimes called the bounded fragment of first-order logic. In [1], it is shown that this fragment is precisely as expressive as the fragment $\mathscr{H}(@, \downarrow)$ of hybrid logic without quantifiers but with the operators $\downarrow$ and @ mentioned in Remark 2.3. Simple syntactic translations between the two formalisms are given. The translations in [1] are for a signature consisting of a single binary relation symbol $R$ (the accessibility relation of unary $\square, \oslash$ ) plus unary relation symbols (which are 
treated as propositional atoms in $\mathscr{H}(@, \downarrow))$. The translation from $\mathscr{H}(@, \downarrow)$ to the bounded fragment generalizes easily to $(k+1)$-ary $R$ (for $k$-ary modalities). The converse translation does not appear to generalize.

The translations still work in the absence of negation and the unary relation symbols. They show that any positive pure $\mathscr{H}(@, \downarrow)$-formula is equivalent to an essentially atomic formula. For binary $R$, the converse holds. To give the idea of this converse translation, the formula

$$
\psi(x)=\forall y(R(x, y) \rightarrow \exists z(R(x, z) \wedge(R(y, z) \vee y=z)))
$$

is easily seen to be equivalent to $\psi^{h}(x)=\square \downarrow_{y} @_{x} \diamond \downarrow_{z}\left(@_{y} \diamond z \vee @_{y} z\right)$. The pseudoequation $\forall x \psi(x)$ is true in a frame $\mathcal{F}$ if and only if the positive pure $\mathcal{H}(@, \downarrow)$ sentence $\downarrow_{x} \psi^{h}(x)$ is valid in $\mathcal{F}$. It follows from this, Remark 3.12, and Theorem 3.13 that in the case of binary $R$ (i.e., unary modalities $-k=1$ ), pure quasipositive sentences are exactly as expressive as positive pure $\mathscr{H}(@, \downarrow)$-sentences.

There are some other relevant translations. We can extend the first-order translation in Remark 3.12 to quasi-positive sentences involving the @ operator. The result is still pseudo-equational, so by Theorem 3.13 , it can be effectively and equivalently rewritten back to a quasi-positive sentence without @. Thus, allowing @ in quasipositive sentences does not change their expressive power. Similarly, the first-order correspondent of any Sahlqvist formula $\sigma$ is pseudo-equational, so we can construct a pure quasi-positive sentence that is valid in the same frames as $\sigma$.

The translations between fragments are summarized in Figure 1.

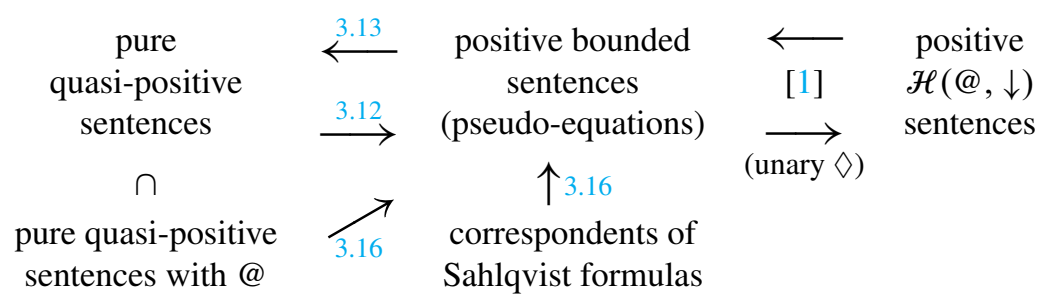

Figure 1 Translations between fragments

\section{Modal Approximations to Hybrid Formulas}

In this section, we will define the "modal approximants" of any hybrid formula $\varphi$. Later, we will show that the validity of a quasi-positive sentence is accurately reflected by validity of its approximants.

An approximant simulates $\varphi$ by substituting modal formulas for the atoms in $\varphi$ and representing nominals by sets in a partition defined by modal formulas. More specifically, an approximant will be with respect to some set $S_{x}$ of $\mathcal{L}$-formulas, for each $x \in \mathcal{V} \cup \ell$. It allocates a conjunction of formulas in $S_{q}$ to each atom $q$. To each nominal $i$, it allocates the conjunction of the formulas in some subset $X$ of $S_{i}$ and the negations of the formulas in $S_{i} \backslash X$. Intuitively, this specifies an equivalence class of the equivalence relation on the worlds of a Kripke model defined by $w \sim w^{\prime}$ if and only if $w, w^{\prime}$ agree on all formulas in $S_{i}$ (it may instead specify the empty 
set of worlds). Quantifiers $\forall i, \exists i$ will be approximated by finite conjunction and disjunction over subsets $X \subseteq S_{i}$.

Formally, the allocation will be done by specifying, for each $x$, a map $\xi_{x}$ from a finite set $\left(S_{x}\right)$ of $\mathcal{L}$-formulas into $\{0,1\}$. An atom $q$ will be allocated the conjunction of the formulas in $\xi_{q}^{-1}$ [1]. A nominal $i$ will be allocated the conjunction of formulas in $\xi_{i}^{-1}[1]$ and the negations of formulas in $\xi_{i}^{-1}[0]$.

For simplicity of notation, we will let $\mathcal{L}$ also denote the set of all (modal) $\mathcal{L}$ formulas over the set $\mathcal{V}$ of atoms. We will write $\varphi, \psi, \ldots$ for (hybrid) $\mathcal{L}^{+}$-formulas, and $\alpha, \beta, \ldots$ for (modal) $\mathcal{L}$-formulas.

\section{Definition 4.1}

1. A display is a sequence $\eta=\left\langle\eta_{x}: x \in \mathcal{V} \cup \ell\right\rangle$ of maps $\eta_{x}: S_{x} \rightarrow\{0,1\}$ where $S_{x}=\operatorname{dom} \eta_{x} \subseteq \mathcal{L}$.

2. A display $\eta$ is said to be finitary if dom $\eta_{x}$ is finite for each $x \in \mathcal{V} \cup \ell$ and full if $\operatorname{dom} \eta_{x}=\mathcal{L}$ for each $x \in \mathcal{V} \cup \ell$.

3. For displays $\xi$, $\eta$, we write $\xi \leq \eta$ if $\xi_{x}$ is a restriction of $\eta_{x}$ (i.e., $\xi_{x} \subseteq \eta_{x}$ ) for every $x \in \mathcal{V} \cup \ell$. (Those familiar with forcing could reasonably prefer it if we wrote $\xi \geq \eta$.)

4. We write $\xi \leq_{\omega} \eta$ if $\xi \leq \eta$ and $\xi$ is finitary.

5. For displays $\xi, \zeta$, and $X \subseteq \mathcal{V} \cup \ell$, we write $\xi={ }_{X} \zeta$ if

(i) $\operatorname{dom} \xi_{x}=\operatorname{dom} \zeta_{x}$ for all $x \in X$, and

(ii) $\xi_{y}=\zeta_{y}$ for all $y \in(\mathcal{V} \cup \ell) \backslash X$.

For $i \in \ell$, we write $\xi={ }_{i} \zeta$ instead of $\xi=\{i\} \zeta$.

From now on, we will try to write $\eta, \theta$ for full displays, and $\xi, \zeta, \delta, \varepsilon$ for finitary displays. We will often use the fact that if $\xi, \zeta \leq_{\omega} \eta$ then there is $\delta$ with $\xi, \zeta \leq \delta \leq_{\omega} \eta$.

Definition 4.2 (Approximants) For each $\mathcal{L}^{+}$-formula $\varphi$ and each finitary display $\xi$, we define a modal $\mathcal{L}$-formula $\varphi_{\xi}$, the $\xi$-approximant of $\varphi$, by induction as follows. Below, $\bigwedge \varnothing$ stands for $T$.

1. For an atom $q$, we let $q_{\xi}=\bigwedge\left\{\alpha \in \operatorname{dom} \xi_{q}: \xi_{q}(\alpha)=1\right\}$.

2. For a nominal $i$, we let $i_{\xi}=\bigwedge\left\{\alpha^{\xi_{i}(\alpha)}: \alpha \in \operatorname{dom} \xi_{i}\right\}$. Here, for an $\mathcal{L}$-formula $\alpha$, we write $\alpha^{1}$ for $\alpha$ and $\alpha^{0}$ for $\neg \alpha$.

3. $\top_{\xi}=\top$ and $\perp_{\xi}=\perp$.

4. $(\neg \varphi)_{\xi}=\neg\left(\varphi_{\xi}\right),(\varphi \wedge \psi)_{\xi}=\varphi_{\xi} \wedge \psi_{\xi}$, and $(\varphi \vee \psi)_{\xi}=\varphi_{\xi} \vee \psi_{\xi}$.

5. $\square\left(\varphi^{1}, \ldots, \varphi^{k}\right)_{\xi}=\square\left(\varphi_{\xi}^{1}, \ldots, \varphi_{\xi}^{k}\right)$, and $\diamond\left(\varphi^{1}, \ldots, \varphi^{k}\right)_{\xi}=\diamond\left(\varphi_{\xi}^{1}, \ldots, \varphi_{\xi}^{k}\right)$.

6. $(\exists i \varphi)_{\xi}=\bigvee_{\zeta=i \xi} \varphi_{\zeta}$, and $(\forall i \varphi)_{\xi}=\bigwedge_{\zeta=i \xi} \varphi_{\zeta}$.

Finally, for any full display $\eta$, we define $\varphi^{\eta}=\left\{\varphi_{\xi}: \xi \leq_{\omega} \eta\right\}$.

Note that $-\xi$ commutes with all operations except quantifiers. We end this section with some basic information on approximants and some examples.

Example 4.3 If $\varphi$ is a modal formula, then a trivial induction will show that for any finitary display $\xi$, the approximant $\varphi_{\xi}$ is just a substitution instance of $\varphi$. Each atom $q$ in $\varphi$ is replaced by the conjunction $q_{\xi}=\bigwedge\left\{\alpha \in \operatorname{dom} \xi_{q}: \xi_{q}(\alpha)=1\right\}$.

Example 4.4 In general, $-\xi$ does not preserve semantic equivalence. For example, the hybrid formulas $\nabla(i \wedge p)$ and $\nabla i \wedge \square(i \rightarrow p)$ are equivalent. But if $\xi$ is a finitary display with $\operatorname{dom} \xi_{i}=\{\alpha\}$ and $\operatorname{dom} \xi_{p}=\{\beta\}$, say, and $\xi_{i}(\alpha)=\xi_{p}(\beta)=1$, then 
$\diamond(i \wedge p)_{\xi}$ is $\diamond(\alpha \wedge \beta)$, while $(\diamond i \wedge \square(i \rightarrow p))_{\xi}$ is $\diamond \alpha \wedge \square(\alpha \rightarrow \beta)$, which is stronger. We will return to this issue in Lemma 5.7.

The next lemma is proved by a simple induction on $\varphi$.

Lemma 4.5 For any $\mathscr{L}^{+}$-formula $\varphi$ and finitary displays $\xi, \zeta$ such that $\xi_{x}=\zeta_{x}$ for each $x \in$ free $(\varphi)$, we have $\varphi_{\zeta}=\varphi_{\zeta}$.

Example 4.6 Let us compute some approximants of the pure quasi-positive sentence $\diamond \exists i \square i$ from Examples 3.4-3.10. Start by letting $S=\{q\} \subseteq \mathcal{L}$, where $q$ is a fixed atom. Let $\xi^{0}, \xi^{1}$ be finitary displays whose $i$-components $\xi_{i}^{0}, \xi_{i}^{1}: S \rightarrow\{0,1\}$ are given by $\xi_{i}^{0}(q)=0$ and $\xi_{i}^{1}(q)=1$. So by definition,

$$
\begin{aligned}
& i_{\xi^{0}}=\bigwedge\left\{\alpha^{\xi_{i}^{0}(\alpha)}: \alpha \in S\right\}=q^{\xi_{i}^{0}(q)}=q^{0}=\neg q, \\
& i_{\xi^{1}}=\bigwedge\left\{\alpha^{\xi_{i}^{1}(\alpha)}: \alpha \in S\right\}=q^{\xi_{i}^{1}(q)}=q^{1}=q .
\end{aligned}
$$

Then for any finitary display $\xi$ with $\operatorname{dom} \xi_{i}=S$ (by Lemma 4.5, the choice of $\xi$ doesn't matter because we are applying it to a pure sentence), we obtain

$$
\begin{aligned}
(\diamond \exists i \square i)_{\xi} & =\diamond(\exists i \square i)_{\xi} \\
& =\diamond \bigvee_{\zeta=i \xi}(\square i)_{\zeta} \\
& =\diamond \bigvee_{l<2}(\square i)_{\xi^{l}} \\
& =\diamond\left(\square\left(i_{\xi^{0}}\right) \vee \square\left(i_{\xi^{1}}\right)\right) \\
& =\diamond(\square \neg q \vee \square q) .
\end{aligned}
$$

This is equivalent to McKinsey's formula $M=\square \diamond q \rightarrow \diamond \square q$ which we saw in the introduction. If we took $S=\left\{q_{1}, \ldots, q_{n}\right\}$ for $n \geq 1$, and for each $X \subseteq S$ put

$$
\tau_{X}=\bigwedge_{q \in X} q \wedge \bigwedge_{q \in S \backslash X} \neg q,
$$

then for a display $\xi$ with $\operatorname{dom} \xi_{i}=S$ we'd essentially get

$$
(\diamond \exists i \square i)_{\xi}=\diamond \bigvee_{X \subseteq S} \square \tau_{X} .
$$

This says that there is a successor all of whose successors agree on the atoms $q_{1}, \ldots, q_{n}$. It is $K$-equivalent to the formula

$$
M_{n}=\diamond \bigwedge_{t=1}^{n}\left(\diamond q_{t} \rightarrow \square q_{t}\right)
$$

given by Lemmon in [30], p. 74, and seen in (2) in the introduction. So each $M_{n}$ has the form $(\diamond \exists i \square i)_{\xi}$ for some finitary display $\xi$. Conversely, for any finitary display $\xi$, the approximant $(\diamond \exists i \square i)_{\xi}$ is $K$-equivalent to a substitution instance of $M_{n}$, where $n=\left|\operatorname{dom} \xi_{i}\right|$. So the $\operatorname{logic} K M^{\infty}$ axiomatized by $\left\{M_{n}: n \geq 1\right\}$ is also axiomatized by $(\diamond \exists i \square i)^{\eta}$ for any full display $\eta$.

Let $\mathcal{K}$ be the class of frames in which "any world sees a world with at most one successor." Lemmon showed that the canonical frame of $K M^{\infty}$ lies in $\mathcal{K}$, and that any frame in $\mathcal{K}$ validates $K M^{\infty}$. So $K M^{\infty}$ is the logic of $\mathcal{K}$; it is canonical and elementarily generated. Clearly, $\mathcal{K}$ is the class of frames that validate $\diamond \exists i \square i$. 
Example 4.7 We briefly do the same for Hughes's logic $K M T$, defined in [24] and mentioned in the introduction. $K M T$ is axiomatized by $\left\{M T_{n}: n \geq 1\right\}$, where

$$
M T_{n}=\diamond\left(\left(\square q_{1} \rightarrow q_{1}\right) \wedge \cdots \wedge\left(\square q_{n} \rightarrow q_{n}\right)\right) .
$$

A similar logic investigated in [23], §4, can be axiomatized by replacing the $\diamond$ by a universal diamond; it can also be handled by the methods below.

Consider the pure quasi-positive sentence

$$
\varphi=\diamond \exists i(i \wedge \diamond i) .
$$

Claim 4.8 KMT is axiomatized by the set $\varphi^{\eta}$ for any full display $\eta$ - again, as $\varphi$ is a pure sentence, there is no dependence here on the choice of $\eta$. We note that identical axioms were obtained in [39], \$3, without using hybrid logic.

Proof of claim For any finitary display $\xi$, it can be checked that

$$
\varphi_{\xi}=\diamond \bigvee_{S \subseteq \operatorname{dom} \xi_{i}}\left(\tau_{S} \wedge \diamond \tau_{S}\right),
$$

where $\tau_{S}=\bigwedge_{\alpha \in S} \alpha \wedge \bigwedge_{\beta \in\left(\operatorname{dom} \xi_{i}\right) \backslash S} \neg \beta$ (as usual, we put $\bigwedge \varnothing=T$ ). Since the $\tau_{S}$ (for $S \subseteq \operatorname{dom} \xi_{i}$ ) are pairwise inconsistent and their disjunction is a tautology, $\varphi_{\xi}$ is $K$-equivalent to $\diamond \bigwedge_{S \subseteq \text { dom } \xi_{i}}\left(\tau_{S} \rightarrow \diamond \tau_{S}\right)$ and so to

$$
\alpha=\diamond \bigwedge_{S \subseteq \text { dom } \xi_{i}}\left(\square \neg \tau_{S} \rightarrow \neg \tau_{S}\right)
$$

This is a substitution instance of $M T_{2^{n}}$, where $n=\left|\operatorname{dom} \xi_{i}\right|$. Conversely, it is not hard to see that $K \vdash \alpha \rightarrow M T_{n}$ in the case where $\operatorname{dom} \xi_{i}=\left\{q_{1}, \ldots, q_{n}\right\}$. The claim is proved.

Let $\mathcal{C}$ be the class consisting of all frames satisfying the first-order condition $\forall x \exists y(R(x, y) \wedge R(y, y))$. In [24], Theorem 1, it is shown that the canonical frame for $K M T$ is in $\mathcal{C}$. Hughes also shows that every frame in $\mathcal{C}$ validates $K M T$. So $K M T$ is the logic of $\mathcal{C}$ and is canonical and elementarily generated. Clearly, $\mathcal{C}$ is the class of frames in which $\varphi$ is valid.

\section{Modal Logics from Quasi-Positive Formulas}

In both the examples just seen, a pure quasi-positive sentence $\varphi$ turned out to characterize (by validity) a class $\mathcal{K}$ of frames containing the canonical frame for the logic axiomatized by $\varphi^{\eta}$. The logic was sound and complete for $\mathcal{K}$. We will prove in this section (Theorem 5.15) that this is always true.

For the whole of this section, we fix a frame $\mathcal{F}=(W, R)$. Given a modal assignment $m: \mathcal{V} \rightarrow \wp(W)$, a formula $\alpha \in \mathscr{L}$, and a set $\Gamma \subseteq \mathcal{L}$, we write

$$
\begin{aligned}
& m(\alpha)=\{w \in W: \mathcal{F}, m, w \models \alpha\}, \\
& m(\Gamma)=\bigcap_{\alpha \in \Gamma} m(\alpha) \quad \text { if } \Gamma \neq \varnothing, \\
& m(\varnothing)=W .
\end{aligned}
$$

We define $h(\varphi)$ and $h(\Gamma)$ similarly, where $h$ is a hybrid assignment into $\mathcal{F}, \varphi$ is an $\mathcal{L}^{+}$-formula, and $\Gamma$ a set of such formulas. 


\subsection{Descriptive assignments}

Definition 5.1 A modal assignment $m: \mathcal{V} \rightarrow \wp(W)$ is said to be descriptive if the following three conditions hold:

separation: If $t \neq u$ in $W$, then there is a modal formula $\alpha$ such that

$$
\mathcal{F}, m, t=\alpha \text { and } \mathcal{F}, m, u \models \neg \alpha \text {. }
$$

tightness: If $t, v_{1}, \ldots, v_{k} \in W$, then $R\left(t, v_{1}, \ldots, v_{k}\right)$ if and only if $\left(\mathcal{F}, m, t \models \diamond\left(\alpha_{1}, \ldots, \alpha_{k}\right)\right.$ whenever $\alpha_{1}, \ldots, \alpha_{k} \in \mathcal{L}$ and $\mathcal{F}, m, v_{l} \models \alpha_{l}$ for each $\left.l=1, \ldots, k\right)$.

saturation: If $\Gamma \subseteq \mathcal{L}$ and $m(\Delta) \neq \varnothing$ for each finite subset $\Delta \subseteq \Gamma$, then $m(\Gamma) \neq \varnothing$.

Of course, there are well-known topological formulations of these conditions; see [33]. This definition is the standard one; note that it concerns only modal formulas.

The next handy lemma will allow us to represent a hybrid assignment's values on nominals by a modal assignment and a full display. If the modal assignment is descriptive, the representation is faithful.

Lemma 5.2 Let $m: \mathcal{V} \rightarrow \wp(W)$ be a modal assignment, and let $w \in W$. Let $i$ be a nominal, and suppose that $\eta$ is a full display satisfying $\eta_{i}(\alpha)=1$ if and only if $w \in m(\alpha)$ for each $\alpha \in \mathcal{L}$. Then,

1. $w \in m\left(i^{\eta}\right)$,

2. if $m$ is descriptive, then $m\left(i^{\eta}\right)=\{w\}$.

Proof Take $\xi \leq_{\omega} \eta$. Then $i_{\xi}=\bigwedge\left\{\alpha^{\xi_{i}(\alpha)}: \alpha \in \operatorname{dom} \xi_{i}\right\}$. For each $\alpha \in \operatorname{dom} \xi_{i}$, if $w \in m(\alpha)$ then $\xi_{i}(\alpha)=1$, so $\alpha$ is a conjunct of $i_{\xi}$. If instead, $w \in m(\neg \alpha)$, then $\xi_{i}(\alpha)=0$, so $\alpha^{0}=\neg \alpha$ is a conjunct of $i_{\xi}$. Hence, $w \in m\left(i_{\xi}\right)$.

Now assume that $m$ is descriptive. By separation, $\left|m\left(i^{\eta}\right)\right| \leq 1$. By this and the first part, we conclude that $m\left(i^{\eta}\right)=\{w\}$.

The obvious analogue of part 1 for finitary displays also holds.

5.2 Monotonicity We would like to show that for any finitary displays $\xi \leq \zeta$ and any quasi-positive $\mathcal{L}^{+}$-formula $\varphi$, we have $\vdash \varphi_{\zeta} \rightarrow \varphi_{\xi}$. The relativized universal quantifiers in $\varphi$ interfere with this aim and cause irritating syntactic issues. But we are able to show it cofinally, which is enough for our later results. We will show that $\vdash \varphi_{\zeta} \rightarrow \varphi_{\xi}$ when $\zeta$ is " $\varphi$-closed." The notion of "closed" will take advantage of the variability of the domains of the maps in a finitary display. Cofinality can be proved for what we call "clean" formulas.

Definition 5.3 Let $\varphi$ be a quasi-positive $\mathcal{L}^{+}$-formula.

1. A finitary display $\zeta$ is said to be $\varphi$-closed if for every subformula of $\varphi$ of the form $\pi^{\diamond}(\top) \wedge \forall i_{1}, \ldots, i_{k}\left(\pi^{\square}\left(\diamond\left(i_{1}, \ldots, i_{k}\right)\right) \rightarrow \psi\right)$, where $\pi^{\diamond}(q)=\diamond^{d_{1}}\left(j_{1} \wedge \diamond^{d_{2}}\left(j_{2} \wedge \cdots \wedge \diamond^{d_{n}}\left(j_{n} \wedge q\right)\right) \cdots\right)$ for some $n \geq 1$, and for every $\zeta^{\prime}=\left\{i_{1}, \ldots, i_{k}\right\} \zeta$, we have $\diamond\left(i_{1}, \ldots, i_{k}\right)_{\zeta^{\prime}} \in \operatorname{dom}\left(\zeta j_{n}\right)$.

2. $\varphi$ is said to be clean if for each subformula $\forall i_{1} \ldots i_{k} \psi$ of $\varphi$, all (atomic) occurrences of $i_{1}, \ldots, i_{k}$ in $\varphi$ are in $\psi$. 


\section{Remark 5.4}

1. Being $\varphi$-closed is only a restriction if $\varphi$ involves universal quantifiers with nonzero $n$ in $\pi$.

2. $\zeta$ being $\varphi$-closed is a property not of the actual maps $\zeta_{i}(i \in \ell)$, but of their domains. If $\zeta={ }_{i} \zeta^{\prime}$ for some nominal $i$, and $\zeta$ is $\varphi$-closed, then so is $\zeta^{\prime}$.

3. Any $\varphi$-closed display is also $\psi$-closed, for any quasi-positive subformula $\psi$ of $\varphi$.

4. By renaming bound nominals, we can easily convert any quasi-positive formula $\varphi$ to a clean quasi-positive formula that is logically equivalent to $\varphi$.

Lemma 5.5 (Cofinality) Let $\varphi$ be a clean quasi-positive formula, let $\eta$ be a full display, and let $\xi \leq_{\omega} \eta$. Then there is a $\varphi$-closed display $\zeta$ with $\xi \leq \zeta \leq_{\omega} \eta$.

Proof Let $V$ be the set of nominals occurring in $\varphi$. Define a graph $G$ on $V$ with edges labeled by subformulas of $\varphi$ as follows. For each subformula

$$
\chi=\pi^{\diamond}(\top) \wedge \forall i_{1}, \ldots, i_{k}\left(\pi^{\square}\left(\diamond\left(i_{1}, \ldots, i_{k}\right)\right) \rightarrow \psi\right)
$$

of $\varphi$, where $\pi^{\diamond}(q)=\diamond^{d_{1}}\left(j_{1} \wedge \diamond^{d_{2}}\left(j_{2} \wedge \cdots \wedge \diamond^{d_{n}}\left(j_{n} \wedge q\right)\right) \cdots\right)$ with $n \geq 1$, include the labeled edges $i_{1} \stackrel{\chi}{\rightarrow} j_{n}, \ldots, i_{k} \stackrel{\chi}{\rightarrow} j_{n}$ in $G$. By Definition 3.3, if $i \stackrel{\chi}{\rightarrow} j$ then $i \neq j$, and clearly, if $i \stackrel{\chi}{\rightarrow} j$ and $i^{\prime} \stackrel{\chi}{\rightarrow} j^{\prime}$ then $j=j^{\prime}$.

Suppose that $i \stackrel{\chi}{\rightarrow} j \stackrel{\chi^{\prime}}{\rightarrow} l$ in $G$. By the above, $\chi \neq \chi^{\prime}$. As $\varphi$ is clean, all occurrences of $j$ in $\varphi$ are in $\chi^{\prime}$. Since $j$ occurs in the $\pi^{\diamond}(T)$-part of $\chi$, it follows that $\chi$ is a proper subformula of $\chi^{\prime}$. Hence, $G$ is acyclic. So we can enumerate $V$ as $\left\{v_{0}, \ldots, v_{m-1}\right\}$ in such a way that if $v_{t} \stackrel{\chi}{\rightarrow} v_{u}$ (for any $\chi$ ) then $t<u$.

Now we define extensions $\xi=\zeta^{0} \leq \zeta^{1} \leq \cdots \leq \zeta^{m}=\zeta \leq_{\omega} \eta$ by induction. In the $t$ th step $(0 \leq t<m)$, write $j$ for $v_{t}$. We define $\zeta_{i}^{t+1}=\zeta_{i}^{t}$ for all $i \in \ell \backslash\{j\}$. As $\zeta^{t+1} \leq_{\omega} \eta$, it remains only to define $\operatorname{dom}\left(\zeta_{j}^{t+1}\right)$ which is done as follows:

1. Include $\operatorname{dom}\left(\zeta_{j}^{t}\right)$ in $\operatorname{dom}\left(\zeta_{j}^{t+1}\right)$.

2. For each subformula $\chi=\pi^{\diamond}(\top) \wedge \forall i_{1}, \ldots, i_{k}\left(\pi^{\square}\left(\diamond\left(i_{1}, \ldots, i_{k}\right)\right) \rightarrow \psi\right)$ of $\varphi$ such that $\left.\pi^{\diamond}(q)=\diamond^{d_{1}}\left(j_{1} \wedge \cdots \wedge \diamond^{d_{n}}(j \wedge q)\right) \cdots\right)$, where $n \geq 1$, and for each $\zeta^{\prime}=\left\{i_{1}, \ldots, i_{k}\right\} \zeta^{t}$, include the formula $\diamond\left(i_{1}, \ldots, i_{k}\right)_{\zeta^{\prime}}$ in $\operatorname{dom}\left(\zeta_{j}^{t+1}\right)$. (There may be none, one, or many such $\chi$ involving $j$ in this way. For each one, we have $i_{1} \stackrel{\chi}{\rightarrow} j, \ldots, i_{k} \stackrel{\chi}{\rightarrow} j$.)

Let us check quickly that $\zeta=\zeta^{m}$ is $\varphi$-closed.

Take a subformula $\chi=\pi^{\diamond}(\top) \wedge \forall i_{1}, \ldots, i_{k}\left(\pi^{\square}\left(\diamond\left(i_{1}, \ldots, i_{k}\right)\right) \rightarrow \psi\right)$ of $\varphi$ such that $\left.\pi^{\diamond}(q)=\diamond^{d_{1}}\left(j_{1} \wedge \cdots \wedge \diamond^{d_{n}}\left(j_{n} \wedge q\right)\right) \cdots\right)$, with $n \geq 1$. Let $t<m$ be the index with $j_{n}=v_{t}$. By construction, $i_{1}, \ldots, i_{k} \in\left\{v_{u}: u<t\right\}$, and so $\operatorname{dom}\left(\zeta_{i_{l}}\right)=\operatorname{dom}\left(\zeta_{i_{l}}^{t}\right)$ for each $l=1, \ldots, k$. Take $\zeta^{\prime}=\left\{i_{1}, \ldots, i_{k}\right\} \zeta$, and let $\zeta^{\dagger} \leq \zeta^{\prime}$ satisfy $\zeta^{\dagger}=\left\{i_{1}, \ldots, i_{k}\right\} \zeta^{t}$. Then also, $\operatorname{dom}\left(\zeta_{i_{l}}^{\prime}\right)=\operatorname{dom}\left(\zeta_{i_{l}}^{\dagger}\right)$, and so $\left(i_{l}\right)_{\zeta^{\prime}}=\left(i_{l}\right)_{\zeta^{\dagger}}$ for each l. It follows from this and the construction that $\diamond\left(i_{1}, \ldots, i_{k}\right)_{\zeta^{\prime}}=\diamond\left(i_{1}, \ldots, i_{k}\right)_{\zeta^{\dagger}}$ $\in \operatorname{dom}\left(\zeta_{j_{n}}^{t+1}\right) \subseteq \operatorname{dom}\left(\zeta_{j_{n}}\right)$, as required.

Definition 5.6 For $1 \leq d \leq k$, we define the binary relation $R^{d}$ on $W$ by

$$
R^{d}(x, y) \Longleftrightarrow \exists z_{1} \ldots z_{k}\left(R\left(x, z_{1}, \ldots, z_{k}\right) \wedge z_{d}=y\right) .
$$

This can be viewed as the "accessibility relation" of $\diamond^{d}$ in $\diamond^{d} \varphi$. 
The next lemma shows how closed displays work. For a nominal $i, \diamond(i \wedge p)$ and $\diamond i \wedge \square(i \rightarrow p)$ are equivalent. We saw in Example 4.4 that when we take approximants, the equivalence can be lost. This problem can be avoided by using closed displays. So to some extent, approximations of nominals with a closed display still behave like real nominals. The lemma is used in Lemma 5.8 and Proposition 5.9.

Lemma 5.7 Let $\chi=\pi^{\diamond}(\top) \wedge \forall i_{1}, \ldots, i_{k}\left(\pi^{\square}\left(\diamond\left(i_{1}, \ldots, i_{k}\right)\right) \rightarrow \varphi\right)$ be a quasipositive formula, where $\pi^{\diamond}(q)=\diamond^{d_{1}}\left(j_{1} \wedge \diamond^{d_{2}}\left(j_{2} \wedge \cdots \wedge \diamond^{d_{n}}\left(j_{n} \wedge q\right)\right) \cdots\right)$ and $n \geq 0$. (If $n=0$, we just have $\pi^{\diamond}(q)=\pi^{\square}(q)=q$.) Then for any $\chi$-closed finitary display $\zeta$, the following modal formula is valid:

$$
\left(\pi^{\diamond}\left(\diamond\left(i_{1}, \ldots, i_{k}\right)\right) \leftrightarrow \pi^{\square}\left(\diamond\left(i_{1}, \ldots, i_{k}\right)\right) \wedge \pi^{\diamond}(\top)\right)_{\zeta} .
$$

Proof The result is trivial if $n=0$. Assume that $n>0$. We leave the easy " $\leftarrow$ " direction as an exercise, since we will not need it. For " $\rightarrow$," as $\mathcal{F}$ is arbitrary, we can work semantically. Take any modal assignment $m$ into $\mathcal{F}$ and any $w \in m\left(\pi^{\diamond}\left(\diamond\left(i_{1}, \ldots, i_{k}\right)\right)_{\zeta}\right)$. For convenience, we also write $w_{0}, u_{0}$ for $w$. Choose $w_{t} \in m\left(\left(j_{t}\right)_{\zeta}\right)$ satisfying $R^{d_{t}}\left(w_{t-1}, w_{t}\right)$ (for each $t$ with $1 \leq t \leq n$ ), and $w_{n} \in m\left(\diamond\left(i_{1}, \ldots, i_{k}\right)_{\zeta}\right)$. From the existence of these $w_{t}$ follows $w \in m\left(\pi^{\diamond}(\top)_{\zeta}\right)$.

It remains to show that $w \in m\left(\pi^{\square}\left(\diamond\left(i_{1}, \ldots, i_{k}\right)\right)_{\zeta}\right)$. As $\zeta$ is $\chi$-closed, $\diamond\left(i_{1}, \ldots, i_{k}\right)_{\zeta} \in \operatorname{dom}\left(\zeta_{j_{n}}\right)$. So either $\diamond\left(i_{1}, \ldots, i_{k}\right)_{\zeta}$ or $\neg \nabla\left(i_{1}, \ldots, i_{k}\right)_{\zeta}$ is a conjunct of $\left(j_{n}\right)_{\zeta}$. As $w_{n} \in m\left(\left(j_{n}\right)_{\zeta}\right) \cap m\left(\diamond\left(i_{1}, \ldots, i_{k}\right)_{\zeta}\right)$, it can only be that $\left(j_{n}\right)_{\zeta} \vdash \diamond\left(i_{1}, \ldots, i_{k}\right)_{\zeta}$. So for any $u_{t} \in m\left(\left(j_{t}\right)_{\zeta}\right)$ satisfying $R^{d_{t}}\left(u_{t-1}, u_{t}\right)$ for $1 \leq t \leq n$, we have $u_{n} \in m\left(\nabla\left(i_{1}, \ldots, i_{k}\right)_{\zeta}\right)$ simply because $u_{n} \in m\left(\left(j_{n}\right)_{\zeta}\right)$. So $w \in m\left(\pi^{\square}\left(\diamond\left(i_{1}, \ldots, i_{k}\right)\right)_{\zeta}\right)$ as required.

Now we can prove the monotonicity lemma.

Lemma 5.8 (Monotonicity) Let $\varphi$ be a quasi-positive formula, and let $\xi \leq \zeta$ be finitary displays such that $\zeta$ is $\varphi$-closed. Then for any modal assignment $m$ into $\mathcal{F}$, we have $m\left(\varphi_{\zeta}\right) \subseteq m\left(\varphi_{\xi}\right)$.

Proof By induction on $\varphi$. If $\varphi$ is atomic, the result is plain because every conjunct of $\varphi_{\xi}$ is also a conjunct of $\varphi_{\zeta}$. The cases $\wedge, \vee, \square, \diamond$ are straightforward as $-{ }_{\xi}$ commutes with these operators. If the result holds for $\varphi, \zeta$ is $\exists i \varphi$-closed, and $w \in m\left((\exists i \varphi)_{\zeta}\right)$, then there is $\zeta^{\prime}={ }_{i} \zeta$ with $w \in m\left(\varphi_{\zeta^{\prime}}\right)$. By Remark 5.4(2) and (3), $\zeta^{\prime}$ is $\varphi$-closed. Let $\xi^{\prime}={ }_{i} \xi$ be the unique display with $\xi^{\prime} \leq \zeta^{\prime}$. Inductively, $m\left(\varphi_{\zeta^{\prime}}\right) \subseteq m\left(\varphi_{\xi^{\prime}}\right)$. So $w \in m\left(\varphi_{\xi^{\prime}}\right) \subseteq m\left((\exists i \varphi)_{\xi}\right)$, as required.

The only complicated case is that of

$$
\begin{aligned}
\chi & =\pi^{\diamond}(\top) \wedge \forall \bar{l}\left(\pi^{\square}(\diamond \bar{l}) \rightarrow \varphi\right), \\
\text { where } \pi^{\diamond}(q) & =\diamond^{d_{1}}\left(j_{1} \wedge \diamond^{d_{2}}\left(j_{2} \wedge \cdots \wedge \diamond^{d_{n}}\left(j_{n} \wedge q\right)\right) \cdots\right) \text { for some } n \geq 0, \\
\text { and } \bar{l} & =\left(i_{1}, \ldots, i_{k}\right)
\end{aligned}
$$

We assume the result for $\varphi$ and prove it for $\chi$. Assume that $\zeta$ is $\chi$-closed. Let $w_{0} \in m\left(\chi_{\zeta}\right)$ be given. Then $w_{0} \in m\left(\pi^{\diamond}(\top)_{\zeta}\right)$, and it follows by earlier cases that $w_{0} \in m\left(\pi^{\diamond}(\top)_{\xi}\right)$.

To show that $w_{0} \in m\left(\forall \bar{l}\left(\pi^{\square}(\diamond \bar{\imath}) \rightarrow \varphi\right) \xi\right)$ as well, assume that $\xi^{\prime}=\left\{i_{1}, \ldots, i_{k}\right\} \xi$ and $w_{0} \in m\left(\pi^{\square}\left(\triangleright_{\bar{\imath}}\right)_{\xi^{\prime}}\right)$. We require

$$
w_{0} \in m\left(\varphi_{\xi^{\prime}}\right) \quad \text { (to be shown). }
$$


We already have $w_{0} \in m\left(\pi^{\diamond}(\top)_{\zeta}\right)$, so we may take $w_{1}, \ldots, w_{n} \in W$ with $R^{d_{t}}\left(w_{t-1}, w_{t}\right)$ and $w_{t} \in m\left(\left(j_{t}\right)_{\zeta}\right)$ for each $1 \leq t \leq n$. As $\xi \leq \zeta$, we have $w_{t} \in m\left(\left(j_{t}\right)_{\xi}\right)=m\left(\left(j_{t}\right)_{\xi^{\prime}}\right)$ for each $1 \leq t \leq n$. Because $w_{0} \in m\left(\pi^{\square}(\diamond \bar{\imath})_{\xi^{\prime}}\right)$, we get $w_{n} \in m\left((\diamond \bar{\imath})_{\xi^{\prime}}\right)$.

Take witnesses $u_{l} \in m\left(\left(i_{l}\right)_{\xi^{\prime}}\right)$ for $1 \leq l \leq k$, with $R\left(w_{n}, u_{1}, \ldots, u_{k}\right)$. Define $\zeta^{\prime}=\left\{i_{1}, \ldots, i_{k}\right\} \zeta$ by $\zeta_{i_{l}}^{\prime}(\alpha)=1$ if and only if $u_{l} \in m(\alpha)$, for each $\alpha \in \operatorname{dom}\left(\zeta_{i_{l}}\right)$ and each $l$. By Lemma 5.2(1), we have $u_{l} \in m\left(\left(i_{l}\right)_{\zeta^{\prime}}\right)$. Hence, $w_{n} \in m\left((\diamond \bar{l})_{\zeta^{\prime}}\right)$, and so $w_{0} \in m\left(\pi^{\diamond}(\nabla \bar{\imath})_{\zeta^{\prime}}\right)$. But by Remark 5.4(2), $\zeta^{\prime}$ is also $\chi$-closed. By Lemma 5.7, $w_{0} \in m\left(\pi^{\square}(\diamond \bar{\imath})_{\zeta^{\prime}}\right)$. As $w_{0} \in m\left(\chi_{\zeta}\right)$, it follows that $w_{0} \in m\left(\left(\pi^{\square}(\diamond \bar{\imath}) \rightarrow \varphi\right)_{\zeta^{\prime}}\right)$, and so $w_{0} \in m\left(\varphi_{\zeta^{\prime}}\right)$.

Because $u_{l} \in m\left(\left(i_{l}\right)_{\zeta^{\prime}}\right) \cap m\left(\left(i_{l}\right)_{\xi^{\prime}}\right)$ for each $l$, we have $\zeta^{\prime} \geq \xi^{\prime}$. Also, $\zeta^{\prime}$ is $\varphi$ closed by Remark 5.4(2) and (3). So inductively, $m\left(\varphi_{\zeta^{\prime}}\right) \subseteq m\left(\varphi_{\xi^{\prime}}\right)$, yielding (10) as required.

So we can always refine a finitary display to a closed one, for which a monotonicity principle holds.

5.3 Persistence of quasi-positive formulas The following is the chief technical result that we need.

Proposition 5.9 Let $\varphi$ be a clean quasi-positive formula. Then for any hybrid assignment $h: \mathcal{V} \cup \ell \rightarrow \wp(W)$ and modal assignment $m: \mathcal{V} \rightarrow \wp(W)$ into $\mathcal{F}$, and any finitary display $\delta$ and full display $\eta$, the following hold:

1. If $h(x) \subseteq m\left(x_{\delta}\right)$ for each $x \in$ free $(\varphi)$, then also $h(\varphi) \subseteq m\left(\varphi_{\delta}\right)$.

2. Hence, if $h(x) \subseteq m\left(x^{\eta}\right)$ for each $x \in$ free $(\varphi)$, then also $h(\varphi) \subseteq m\left(\varphi^{\eta}\right)$.

3. If $m$ is descriptive and $m\left(x^{\eta}\right) \subseteq h(x)$ for each $x \in$ free $(\varphi)$, then $m\left(\varphi^{\eta}\right) \subseteq h(\varphi)$.

Proof Part 2 is immediate from part 1. To see this, we note that for each $x \in$ free $\varphi$, if $h(x) \subseteq m\left(x^{\eta}\right)$ then $h(x) \subseteq m\left(x_{\delta}\right)$ for each $\delta \leq_{\omega} \eta$. By part $1, h(\varphi) \subseteq m\left(\varphi_{\delta}\right)$ for each such $\delta$, so that $h(\varphi) \subseteq \bigcap\left\{m\left(\varphi_{\delta}\right): \delta \leq_{\omega} \eta\right\}=m\left(\varphi^{\eta}\right)$.

We prove parts 1 and 3 together by induction on $\varphi$. If $\varphi \in \mathcal{V} \cup \ell$, we are given the results, and the cases $T$ and $\perp$ are trivial. Inductively assume the lemma for the quasi-positive formulas $\varphi, \psi, \varphi^{1}, \ldots, \varphi^{k}$. We consider the formulas made from them by cases. All but the quantifier cases are pretty much the same as in the proof of the classical Sahlqvist theorem; see, for example, [4], and [3].

Case $\varphi \vee \psi \quad$ Assume that $h(x) \subseteq m\left(x_{\delta}\right)$ for each $x \in$ free $(\varphi \vee \psi)$. Then $h(x) \subseteq m\left(x_{\delta}\right)$ for each $x \in$ free $(\varphi)$, so inductively, $h(\varphi) \subseteq m\left(\varphi_{\delta}\right)$. Similarly, $h(\psi) \subseteq m\left(\psi_{\delta}\right)$. Hence, $h(\varphi \vee \psi)=h(\varphi) \cup h(\psi) \subseteq m\left(\varphi_{\delta}\right) \cup m\left(\psi_{\delta}\right)=m\left(\varphi_{\delta} \vee \psi_{\delta}\right)$ $=m\left((\varphi \vee \psi)_{\delta}\right)$.

Conversely, if $m\left(x^{\eta}\right) \subseteq h(x)$ for each $x \in$ free $(\varphi \vee \psi)$, then

$$
\begin{aligned}
m\left((\varphi \vee \psi)^{\eta}\right) & =m\left(\left\{(\varphi \vee \psi)_{\xi}: \xi \leq_{\omega} \eta\right\}\right) & & \text { (definition of } \left.-{ }^{\eta}\right) \\
& =m\left(\left\{\varphi_{\xi} \vee \psi_{\xi}: \xi \leq_{\omega} \eta\right\}\right) & & \text { (definition of }-\xi) \\
& =m\left(\left\{\varphi_{\xi}: \xi \leq_{\omega} \eta\right\}\right) \cup m\left(\left\{\psi_{\zeta}: \zeta \leq_{\omega} \eta\right\}\right) & & \text { (see }(*) \text { below) } \\
& =m\left(\varphi^{\eta}\right) \cup m\left(\psi^{\eta}\right) & & \text { (definition of }-{ }^{\eta} \text { ) } \\
& \subseteq h(\varphi) \cup h(\psi) & & \text { (inductively) } \\
& =h(\varphi \vee \psi) . & &
\end{aligned}
$$

Let us check that the equality $(*)$ is true. The inclusion " $\supseteq$ " is clear (and we don't need it). For the converse, let $w \in W$ and suppose that $w \notin m\left(\left\{\varphi_{\xi}: \xi \leq{ }_{\omega} \eta\right\}\right) \cup$ 
$m\left(\left\{\psi_{\zeta}: \zeta \leq_{\omega} \eta\right\}\right)$. Then there are $\xi, \zeta \leq_{\omega} \eta$ with $w \notin m\left(\varphi_{\xi}\right)$ and $w \notin m\left(\psi_{\zeta}\right)$. Pick $\varepsilon \leq_{\omega} \eta$ with $\xi, \zeta \leq \varepsilon$. As $\varphi \vee \psi$ is assumed clean, by Lemma 5.5 we can assume that $\varepsilon$ is $(\varphi \vee \psi)$-closed. Hence it is $\varphi$-closed and $\psi$-closed. By Lemma 5.8, $w \notin m\left(\varphi_{\varepsilon}\right)$ and $w \notin m\left(\psi_{\varepsilon}\right)$. So $w \notin m\left(\varphi_{\varepsilon}\right) \cup m\left(\psi_{\varepsilon}\right)=m\left(\varphi_{\varepsilon} \vee \psi_{\varepsilon}\right)$. This shows that $w \notin m\left(\left\{\varphi_{\xi} \vee \psi_{\xi}: \xi \leq_{\omega} \eta\right\}\right)$, as required.

Case $\varphi \wedge \psi \quad$ This is similar and, in fact, easier. We leave it to the reader.

Case $\square\left(\varphi^{1}, \ldots, \varphi^{k}\right) \quad$ To reduce clutter, it will be implicit that $l$ ranges over $1, \ldots, k$. Assume first that $h(x) \subseteq m\left(x_{\delta}\right)$ for each $x \in$ free $\left(\square\left(\varphi^{1}, \ldots, \varphi^{k}\right)\right)$. So the same is true for each $\varphi^{l}$. Inductively, $h\left(\varphi^{l}\right) \subseteq m\left(\varphi_{\delta}^{l}\right)$ for each $l$. So for any $w \in h\left(\square\left(\varphi^{1}, \ldots, \varphi^{k}\right)\right)$ and $v_{1}, \ldots, v_{k} \in W$, if $R\left(w, v_{1}, \ldots, v_{k}\right)$ then for some $l$ we have $v_{l} \in h\left(\varphi^{l}\right) \subseteq m\left(\varphi_{\delta}^{l}\right)$. This implies that $w \in m\left(\square\left(\varphi_{\delta}^{1}, \ldots, \varphi_{\delta}^{k}\right)\right)=m\left(\square\left(\varphi^{1}\right.\right.$, $\left.\ldots, \varphi^{k}\right)_{\delta}$ ), as required.

Conversely, suppose that $m\left(x^{\eta}\right) \subseteq h(x)$ for $x \in$ free $\left(\square\left(\varphi^{1}, \ldots, \varphi^{k}\right)\right)$. Pick arbitrary $w \in m\left(\square\left(\varphi^{1}, \ldots, \varphi^{k}\right)^{\eta}\right)$. Then $w \in m\left(\square\left(\varphi_{\xi}^{1}, \ldots, \varphi_{\xi}^{k}\right)\right)$ for all $\xi \leq_{\omega} \eta$.

We claim that, for all $v_{1}, \ldots, v_{k} \in W$ with $R\left(w, v_{1}, \ldots, v_{k}\right)$, there is $l$ with $v_{l} \in m\left(\varphi_{\xi}^{l}\right)$ for all $\xi \leq_{\omega} \eta$. For if not, then there are $v_{1}, \ldots, v_{k} \in W$ with $R\left(w, v_{1}, \ldots, v_{k}\right)$ and such that for each $l$ there is $\xi^{l} \leq_{\omega} \eta$ with $v_{l} \notin m\left(\varphi_{\xi l}^{l}\right)$. Pick $\zeta \leq_{\omega} \eta$ with $\xi^{1}, \ldots, \xi^{k} \leq \zeta$. By Lemma 5.5 , we may assume that $\zeta$ is $\square\left(\varphi^{1}, \ldots\right.$, $\left.\varphi^{k}\right)$-closed, and hence $\varphi^{l}$-closed for each $l$. By Lemma 5.8, $v_{l} \notin m\left(\varphi_{\zeta}^{l}\right)$ for each $l$. This contradicts $w \in m\left(\square\left(\varphi_{\zeta}^{1}, \ldots, \varphi_{\zeta}^{k}\right)\right)$. The claim is proved.

By the claim, for all $v_{1}, \ldots, v_{k} \in W$ with $R\left(w, v_{1}, \ldots, v_{k}\right)$, there is $l$ with $v_{l} \in m\left(\left(\varphi^{l}\right)^{\eta}\right)$. Inductively, $v_{l} \in h\left(\varphi^{l}\right)$. So $w \in h\left(\square\left(\varphi^{1}, \ldots, \varphi^{k}\right)\right)$.

Case $\diamond\left(\varphi^{1}, \ldots, \varphi^{k}\right) \quad$ Again it will be implicit that $l$ ranges over $1, \ldots, k$. First assume that $h(x) \subseteq m\left(x_{\delta}\right)$ for each $x \in$ free $\left(\diamond\left(\varphi^{1}, \ldots, \varphi^{k}\right)\right)$. So the same holds for each $\varphi^{l}$, and inductively, $h\left(\varphi^{l}\right) \subseteq m\left(\varphi_{\delta}^{l}\right)$. Let $w \in h\left(\diamond\left(\varphi^{1}, \ldots, \varphi^{k}\right)\right)$ be arbitrary. So there are $v_{1}, \ldots, v_{k} \in W$ with $R\left(w, v_{1}, \ldots, v_{k}\right)$ and $v_{l} \in h\left(\varphi^{l}\right)$ for each $l$. Hence, $v_{l} \in m\left(\varphi_{\delta}^{l}\right)$ for each $l$, and by semantics of $\diamond$ we obtain $w \in m\left(\diamond\left(\varphi_{\delta}^{1}, \ldots\right.\right.$, $\left.\left.\varphi_{\delta}^{k}\right)\right)=m\left(\diamond\left(\varphi^{1}, \ldots, \varphi^{k}\right)_{\delta}\right)$.

The converse follows easily from Esakia's well-known lemma on distribution of $\diamond$ over directed intersections; see, for example, [4], Lemma 10.27, for the unary case $(k=1)$ and [19], Lemma 53, for the polyadic case. We give a proof so that our paper is more self-contained and to introduce some machinery that will be needed in the next case. Consider the set

$$
\Xi=\left\{\zeta: \zeta \leq_{\omega} \eta, \zeta \text { is } \diamond\left(\varphi^{1}, \ldots, \varphi^{k}\right) \text {-closed }\right\} .
$$

We will build a set ultrafilter on this set-that is, an ultrafilter of the Boolean algebra $(\wp(\Xi), \cup, \backslash)$. For any $\xi \leq_{\omega} \eta$, let $\xi \uparrow=\{\zeta \in \Xi: \xi \leq \zeta\}$. Then, by Lemma 5.5, the intersection of any finite number of sets of the form $\xi \uparrow$ (for $\xi \leq_{\omega} \eta$ ) is nonempty. By the Boolean prime ideal theorem ([5], 4.1.3), there is an ultrafilter $\mathscr{D}$ on $\Xi$ containing $\xi \uparrow$ for each $\xi \leq_{\omega} \eta$.

Moving toward the proof of the converse now, assume that $m$ is descriptive, and let $w \in m\left(\diamond\left(\varphi^{1}, \ldots, \varphi^{k}\right)^{\eta}\right)$ be arbitrary. Then $w \in m\left(\diamond\left(\varphi^{1}, \ldots, \varphi^{k}\right)_{\xi}\right)=m\left(\diamond\left(\varphi_{\xi}^{1}\right.\right.$, $\left.\ldots, \varphi_{\xi}^{k}\right)$ ) for each $\xi \leq_{\omega} \eta$. So for each $\xi$, there are points $v_{l}^{\xi} \in m\left(\varphi_{\xi}^{l}\right)$ for each $l$, with $R\left(w, v_{1}^{\xi}, \ldots, v_{k}^{\xi}\right)$. 
We are going to use saturation to eliminate the dependence of the $v_{l}^{\xi}$ on $\xi$. The ultrafilter $\mathscr{D}$ will make some choices for us, and it plays the role that Tychonoff's theorem plays in topological proofs of Esakia's lemma. For each $l$, let

$$
\begin{aligned}
{[\alpha]_{l} } & =\left\{\zeta \in \Xi: v_{l}^{\zeta} \in m(\alpha)\right\}, \text { for each } \alpha \in \mathscr{L}, \\
\Gamma_{l} & =\left\{\alpha \in \mathscr{L}:[\alpha]_{l} \in \mathscr{D}\right\} .
\end{aligned}
$$

$\Gamma_{l}$ is simply the set of $\mathscr{L}$-formulas that hold at a "majority" (as determined by $\mathscr{D})$ of the $v_{l}^{\zeta}$. Let $\alpha_{1}, \ldots, \alpha_{n} \in \Gamma_{l}$, for any finite $n$. As $\mathcal{D}$ is an ultrafilter, $\left[\alpha_{1}\right]_{l} \cap \cdots \cap\left[\alpha_{n}\right]_{l} \in \mathcal{D}$, so certainly there is $\zeta \in\left[\alpha_{1}\right]_{l} \cap \cdots \cap\left[\alpha_{n}\right]_{l}$. Then $v_{l}^{\zeta} \in m\left(\alpha_{1}\right) \cap \cdots \cap m\left(\alpha_{n}\right)$. It follows that $m(\Delta) \neq \varnothing$ for every finite $\Delta \subseteq \Gamma_{l}$. So by saturation, we may pick $v_{l} \in m\left(\Gamma_{l}\right)$, for each $l$.

Note that

$$
\Gamma_{l}=\left\{\alpha \in \mathcal{L}: \mathcal{F}, m, v_{l} \models \alpha\right\} .
$$

This follows from the fact that for any $\alpha$, the sets $[\alpha]_{l}$ and $[\neg \alpha]_{l}$ are disjoint and $[\alpha]_{l} \cup[\neg \alpha]_{l}=\Xi \in \mathscr{D}$. So exactly one of $[\alpha]_{l},[\neg \alpha]_{l}$ is in $\mathscr{D}$, and exactly one of $\alpha, \neg \alpha$ is in $\Gamma_{l}$. Since $v_{l}$ satisfies all the formulas in $\Gamma_{l}$, it satisfies precisely these formulas.

We next show that $v_{l} \in m\left(\left(\varphi^{l}\right)^{\eta}\right)$ for each $l$. Pick any $\xi \leq_{\omega} \eta$. By Lemma 5.8, if $\zeta \in \xi \uparrow$ then $v_{l}^{\zeta} \in m\left(\varphi_{\zeta}^{l}\right) \subseteq m\left(\varphi_{\xi}^{l}\right)$. So $\xi \uparrow \subseteq\left[\varphi_{\xi}^{l}\right]_{l}$. By choice of $\mathcal{D}$, this implies that $\left[\varphi_{\xi}^{l}\right]_{l} \in \mathscr{D}$, so that $\varphi_{\xi}^{l} \in \Gamma_{l}$, and $v_{l} \in m\left(\varphi_{\xi}^{l}\right)$. This holds for all $\xi \leq_{\omega} \eta$, so $v_{l} \in m\left(\left(\varphi^{l}\right)^{\eta}\right)$ as required.

We check finally that $R\left(w, v_{1}, \ldots, v_{k}\right)$. For this we use the tightness property of $m$. Let $\alpha_{1}, \ldots, \alpha_{k} \in \mathcal{L}$. Suppose that $\mathcal{F}, m, v_{l} \models \alpha_{l}$ for each $l$. Then by (12), $\alpha_{l} \in \Gamma_{l}$, so $\left[\alpha_{l}\right]_{l} \in \mathcal{D}$ for each $l$. Hence there is $\zeta \in\left[\alpha_{1}\right]_{1} \cap \cdots \cap\left[\alpha_{k}\right]_{k}$. Then $\mathcal{F}, m, v_{l}^{\zeta} \models \alpha_{l}$ for each $l$. Since $R\left(w, v_{1}^{\zeta}, \ldots, v_{k}^{\zeta}\right)$, tightness of $m$ yields $\mathcal{F}, m, w \models \diamond\left(\alpha_{1}, \ldots, \alpha_{k}\right)$. This holds for all $\alpha_{1}, \ldots, \alpha_{k}$, so the other direction of tightness now gives $R\left(w, v_{1}, \ldots, v_{k}\right)$, as required.

To sum up, if $m$ is descriptive and $w \in m\left(\diamond\left(\varphi^{1}, \ldots, \varphi^{k}\right)^{\eta}\right)$, then there are $v_{l} \in m\left(\left(\varphi^{l}\right)^{\eta}\right)$ for each $l$, with $R\left(w, v_{1}, \ldots, v_{k}\right)$. Suppose $m\left(x^{\eta}\right) \subseteq h(x)$ for each $x \in$ free $\left(\diamond\left(\varphi^{1}, \ldots, \varphi^{k}\right)\right)$. Then the same holds for each $x \in$ free $\left(\varphi^{l}\right)$, so inductively, $m\left(\left(\varphi^{l}\right)^{\eta}\right) \subseteq h\left(\varphi^{l}\right)$. Hence, $v_{l} \in h\left(\varphi^{l}\right)$ for each $l$, and it follows that $w \in h\left(\diamond\left(\varphi^{1}\right.\right.$, $\left.\left.\ldots, \varphi^{k}\right)\right)$, as required.

Case $\exists i \varphi \quad$ Fix $h$, and let $G$ be the set of all hybrid assignments $g$ into $\mathcal{F}$ satisfying $g(x)=h(x)$ for all $x \in \mathcal{V} \cup \ell \backslash\{i\}$. Then by definition of the semantics,

$$
h(\exists i \varphi)=\bigcup_{g \in G} g(\varphi) .
$$

First, suppose that $h(x) \subseteq m\left(x_{\delta}\right)$ for each $x \in$ free $(\exists i \varphi)$. Pick any $g \in G$; by (13), it is enough if we show that $g(\varphi) \subseteq m\left((\exists i \varphi)_{\delta}\right)$.

Define $\varepsilon={ }_{i} \delta$ by $\varepsilon(\alpha)=1$ if and only if $g(i) \subseteq m(\alpha)$, for each $\alpha \in \operatorname{dom} \delta_{i}$. By Lemma 5.2(1), we have $g(i) \subseteq m\left(i_{\varepsilon}\right)$. Now $g \in G$ and $\varepsilon={ }_{i} \delta$. So for every $x \in$ free $(\exists i \varphi)$, we have $g(x)=h(x) \subseteq m\left(x_{\delta}\right)=m\left(x_{\varepsilon}\right)$. As free $(\varphi)=$ free $(\exists i \varphi) \cup\{i\}$, we obtain $g(x) \subseteq m\left(x_{\varepsilon}\right)$ for each $x \in$ free $(\varphi)$. Using the inductive hypothesis, we obtain the required inclusion

$$
g(\varphi) \subseteq m\left(\varphi_{\varepsilon}\right) \subseteq m\left(\bigvee_{\zeta=i} \varphi_{\zeta}\right)=m\left((\exists i \varphi)_{\delta}\right)
$$


The converse is proved rather as in the case of $\nabla$. Let $\Xi=\left\{\zeta: \zeta \leq_{\omega} \eta, \zeta\right.$ is $\exists i \varphi$-closed $\}$, and let $\mathscr{D}$ be an ultrafilter on $\Xi$ containing $\xi \uparrow=\{\zeta \in \Xi: \xi \leq \zeta\}$, for $\xi \leq_{\omega} \eta$. Assume that $m$ is descriptive and that $m\left(x^{\eta}\right) \subseteq h(x)$ for each $x \in$ free $(\exists i \varphi)$. Take any $w \in m\left((\exists i \varphi)^{\eta}\right)$. We will show that $w \in h(\exists i \varphi)$.

By definition of $(\exists i \varphi)^{\eta}$,

$$
m\left((\exists i \varphi)^{\eta}\right)=m\left(\left\{\bigvee_{\zeta=i \xi} \varphi_{\zeta}: \xi \leq_{\omega} \eta\right\}\right) .
$$

So for all $\xi \leq_{\omega} \eta$ there is some $\xi^{*}=_{i} \xi$ with $w \in m\left(\varphi_{\xi^{*}}\right)$. For each $\alpha \in \mathscr{L}$, put

$$
\begin{aligned}
& \alpha^{+}=\left\{\zeta \in \Xi: \alpha \in \operatorname{dom} \zeta_{i}, \zeta_{i}^{*}(\alpha)=1\right\}, \\
& \alpha^{-}=\left\{\zeta \in \Xi: \alpha \in \operatorname{dom} \zeta_{i}, \zeta_{i}^{*}(\alpha)=0\right\} .
\end{aligned}
$$

Define $\theta={ }_{i} \eta$ by,

$$
\text { for each } \alpha \in \mathcal{L}, \quad \theta_{i}(\alpha)=1 \Longleftrightarrow \alpha^{+} \in \mathcal{D} .
$$

As in the ' $\nabla$ ' case, this is saying that $\theta$ agrees with the majority of $\zeta^{*}$.

Claim 5.10 $w \in m\left(\varphi^{\theta}\right)$.

Proof of claim Let $\varepsilon \leq_{\omega} \theta$; we show that $w \in m\left(\varphi_{\varepsilon}\right)$. Let $\varepsilon^{\prime}={ }_{i} \varepsilon$ satisfy $\varepsilon^{\prime} \leq_{\omega} \eta$. Then $\varepsilon^{\prime} \uparrow \in \mathscr{D}$. If $\alpha \in \operatorname{dom} \varepsilon_{i}$ then, clearly, $\alpha^{+} \cup \alpha^{-} \supseteq \varepsilon^{\prime} \uparrow$, so $\alpha^{+} \cup \alpha^{-} \in \mathscr{D}$. The sets $\alpha^{+}, \alpha^{-}$are disjoint, so exactly one of them is in $\mathscr{D}$. Since dom $\varepsilon_{i}$ is finite, we may find $S \in \mathscr{D}$ such that $S \subseteq \varepsilon^{\prime} \uparrow$ and with the property that

$$
S \subseteq \alpha^{+} \text {or } S \subseteq \alpha^{-}, \quad \text { for each } \alpha \in \operatorname{dom} \varepsilon_{i} .
$$

Pick any $\zeta \in S$. Then $\operatorname{dom} \varepsilon_{i} \subseteq \operatorname{dom} \zeta_{i}$. By (14), (16), and (15), for every $\alpha \in \operatorname{dom} \varepsilon_{i}$ we have

$$
\zeta_{i}^{*}(\alpha)=1 \Longleftrightarrow \zeta \in \alpha^{+} \Longleftrightarrow S \subseteq \alpha^{+} \Longleftrightarrow \alpha^{+} \in \mathbb{D} \Longleftrightarrow \theta_{i}(\alpha)=1 .
$$

So $\theta_{i}(\alpha)=\varepsilon_{i}(\alpha)=\zeta_{i}^{*}(\alpha)$ for each $\alpha \in \operatorname{dom} \varepsilon_{i}$. Now recall that $\zeta \in S \subseteq \varepsilon^{\prime} \uparrow$. So $\zeta \geq \varepsilon^{\prime}$. But $\zeta^{*}={ }_{i} \zeta$ and $\theta={ }_{i} \eta$, so it follows that $\zeta^{*} \geq \varepsilon$. We know that $w \in m\left(\varphi_{\zeta^{*}}\right)$. By Remark 5.4(2) and (3), $\zeta^{*}$ is $\varphi$-closed. So, by Lemma 5.8, $m\left(\varphi_{\zeta^{*}}\right) \subseteq m\left(\varphi_{\varepsilon}\right)$, and so $w \in m\left(\varphi_{\varepsilon}\right)$. As $\varepsilon \leq_{\omega} \theta$ was arbitrary, $w \in m\left(\varphi^{\theta}\right)$, as claimed.

By separation, $\left|m\left(i^{\theta}\right)\right| \leq 1$. So we may choose $g \in G$ with $g(i) \supseteq m\left(i^{\theta}\right)$. We assumed that $m\left(x^{\eta}\right) \subseteq h(x)$ for each $x \in$ free $(\exists i \varphi)$. As $\theta=i \eta$ and $g \in G$, it follows that $m\left(x^{\theta}\right) \subseteq g(x)$ for all $x \in$ free $(\varphi)$. By the claim, the inductive hypothesis, and (13), we now obtain $w \in m\left(\varphi^{\theta}\right) \subseteq g(\varphi) \subseteq h(\exists i \varphi)$ as required.

Case of relativized $\forall i \quad$ Suppose that $n<\omega, 1 \leq d_{1}, \ldots, d_{n} \leq k, j_{1}, \ldots, j_{n} \in \ell$, and $i_{1}, \ldots, i_{k} \in \ell \backslash\left\{j_{1}, \ldots, j_{n}\right\}$. Let

$$
\begin{aligned}
\pi^{\diamond}(q) & =\diamond^{d_{1}}\left(j_{1} \wedge \diamond^{d_{2}}\left(j_{2} \wedge \cdots \wedge \diamond^{d_{n}}\left(j_{n} \wedge q\right)\right) \cdots\right), \\
\pi & =\pi^{\diamond}(T), \\
\rho & =\pi \square\left(\diamond\left(i_{1}, \ldots, i_{k}\right)\right) \\
\chi & =\pi \wedge \forall i_{1} \ldots i_{k}(\rho \rightarrow \varphi) .
\end{aligned}
$$

If $n=0$, we have $\pi=\top$ and $\rho=\diamond\left(i_{1}, \ldots, i_{k}\right)$. Suppose that

$$
h\left(j_{t}\right)=\left\{w_{t}\right\} \quad \text { for } 1 \leq t \leq n .
$$


We prove the result for $\chi$. First assume that $h(x) \subseteq m\left(x_{\delta}\right)$ for all $x \in$ free $(\chi)$. We require $h(\chi) \subseteq m\left(\chi_{\delta}\right)$. We have $h(x) \subseteq m\left(x_{\delta}\right)$ for all $x \in$ free $\pi$, so by the preceding cases, $h(\pi) \subseteq m\left(\pi_{\delta}\right)$. Let $w=w_{0} \in h(\chi)$. Take $\varepsilon=\left\{i_{1}, \ldots, i_{k}\right\} \delta$, and suppose that $w \in m\left(\rho_{\varepsilon}\right)$. We need to show that $w \in m\left(\varphi_{\varepsilon}\right)$.

Because $w \in h(\chi) \subseteq h(\pi)$, we have $R^{d_{t}}\left(w_{t-1}, w_{t}\right)$ for $1 \leq t \leq n$. By our original assumption and the fact that $\varepsilon=\left\{i_{1}, \ldots, i_{k}\right\} \delta$, we have $h\left(j_{t}\right) \subseteq m\left(\left(j_{t}\right)_{\delta}\right)=m\left(\left(j_{t}\right)_{\varepsilon}\right)$, and so $w_{t} \in m\left(\left(j_{t}\right)_{\varepsilon}\right)$ for each $t$. As $w \in m\left(\rho_{\varepsilon}\right)$, we see that $w_{n} \in m\left(\diamond\left(i_{1}, \ldots, i_{k}\right)_{\varepsilon}\right)$ (note that this is true even if $n=0$ ). So we may find $u_{l} \in m\left(\left(i_{l}\right)_{\varepsilon}\right)$ for $l=1, \ldots, k$, with $R\left(w_{n}, u_{1}, \ldots, u_{k}\right)$.

Define a hybrid assignment $g$ into $\mathcal{F}$, agreeing with $h$ except on $i_{1}, \ldots, i_{k}$, and with $g\left(i_{l}\right)=\left\{u_{l}\right\}$ for each $l$. Then it is plain that $w \in g(\rho)$. Since $w \in h(\chi)$, we see that $w \in g(\varphi)$. Now by assumption and the choice of the $u_{l}$, we have $g(x) \subseteq m\left(x_{\varepsilon}\right)$ for each $x \in$ free $(\varphi)$. So inductively, $g(\varphi) \subseteq m\left(\varphi_{\varepsilon}\right)$, and we obtain $w \in m\left(\varphi_{\varepsilon}\right)$ as required. We make some comments on this argument in Remark 5.11 below.

The converse is very similar to the proof of Lemma 5.8. Assume that $m$ is descriptive and that $m\left(x^{\eta}\right) \subseteq h(x)$ for all $x \in$ free $(\chi)$. Suppose that $w \in m\left(\chi^{\eta}\right)$. By distributing the infinite intersection here over the conjunction in $\chi$, we get $w \in m\left(\pi^{\eta}\right)$. We have $m\left(j_{t}^{\eta}\right) \subseteq h\left(j_{t}\right)$ for $t=1, \ldots, n$, so by earlier cases,

$$
w \in h(\pi)=h\left(\pi^{\diamond}(\top)\right) .
$$

To show that $w \in h\left(\forall i_{1} \ldots i_{k}(\rho \rightarrow \varphi)\right)$ as well, let $g$ be an arbitrary hybrid assignment into $\mathcal{F}$ that agrees with $h$ except perhaps on $i_{1}, \ldots, i_{k}$, and with $w \in g(\rho)$. We require that $w \in g(\varphi)$.

Using Lemma 5.2, pick a full display $\theta=\left\{i_{1}, \ldots, i_{k}\right\} \eta$ such that $m\left(i_{l}^{\theta}\right)=g\left(i_{l}\right)$ for each $l$. Then $m\left(x^{\theta}\right) \subseteq g(x)$ for each $x \in$ free $(\varphi)$. Inductively, $m\left(\varphi^{\theta}\right) \subseteq g(\varphi)$. So if we can show that $w \in m\left(\varphi^{\theta}\right)$, it will follow that $w \in g(\varphi)$ as required.

From (18), it is clear that $w \in g\left(\pi^{\diamond}(\top)\right)$. We assumed that $w \in g(\rho)-$ that is, $w \in g\left(\pi \square\left(\diamond\left(i_{1}, \ldots, i_{k}\right)\right)\right)$. So by ordinary hybrid evaluation, $w \in$ $g\left(\pi^{\diamond}\left(\diamond\left(i_{1}, \ldots, i_{k}\right)\right)\right)$. This formula is quasi-positive. We check that $g(x) \subseteq m\left(x^{\theta}\right)$ for all free nominals $x$ in it. For nominals $i_{l}$, it holds by choice of $\theta$. For nominals $j_{t}$, since $w \in m\left(\pi^{\eta}\right)$, it follows that $m\left(\left(j_{t}\right)_{\xi}\right) \neq \varnothing$ for every $\xi \leq_{\omega} \eta$. So, clearly, $m(\Delta) \neq \varnothing$ for every finite $\Delta \subseteq j_{t}^{\eta}$. By saturation, $m\left(j_{t}^{\eta}\right) \neq \varnothing$. Since we assumed that $m\left(j_{t}^{\eta}\right) \subseteq h\left(j_{t}\right)=\left\{w_{t}\right\}$, we obtain $m\left(j_{t}^{\eta}\right)=h\left(j_{t}\right)$. Hence, $g\left(j_{t}\right)=h\left(j_{t}\right) \subseteq m\left(j_{t}^{\eta}\right)=m\left(j_{t}^{\theta}\right)$. So by earlier cases of part 2 of the proposition, $w \in m\left(\pi^{\diamond}\left(\diamond\left(i_{1}, \ldots, i_{k}\right)\right)^{\theta}\right)$.

Let $\zeta \leq_{\omega} \theta$ be $\chi$-closed. Then $w \in m\left(\pi^{\diamond}\left(\diamond\left(i_{1}, \ldots, i_{k}\right)\right)_{\zeta}\right)$. By Lemma 5.7, $w \in m\left(\pi^{\square}\left(\diamond\left(i_{1}, \ldots, i_{k}\right)\right)_{\zeta}\right)$ - that is, $w \in m\left(\rho_{\zeta}\right)$. But also, $w \in m\left(\rho_{\zeta} \rightarrow \varphi_{\zeta}\right)$. (This is because if we pick $\xi \leq_{\omega} \eta$ with $\xi=\left\{i_{1}, \ldots, i_{k}\right\} \zeta$, we have $w \in m\left(\chi^{\eta}\right) \subseteq m(\chi \xi)$ $\subseteq m\left(\forall i_{1} \ldots i_{k}(\rho \rightarrow \varphi)_{\xi}\right) \subseteq m\left((\rho \rightarrow \varphi)_{\zeta}\right)$.) Combining these gives $w \in m\left(\varphi_{\zeta}\right)$. As this holds for all $\chi$-closed $\zeta \leq_{\omega} \theta$, we easily deduce from Lemmas 5.5 and 5.8 that $w \in m\left(\varphi^{\theta}\right)$ as desired. This completes the proof of Proposition 5.9.

Remark 5.11 The rather elaborate form of relativized universal quantification in quasi-positive formulas makes life complicated. Here we will look at the definition in more detail and see that simpler alternatives would not allow Proposition 5.9 to be proved.

The real role of the conjunct $\pi$ of $\chi$ in the soundness part of the $\forall$-case in Proposition 5.9 is to ensure that $m\left(\left(i_{l}\right)_{\varepsilon}\right) \neq \varnothing$ for each $l$. Then we can choose $g$ with 
$g\left(i_{l}\right) \subseteq m\left(\left(i_{l}\right)_{\varepsilon}\right)$ and apply the inductive hypothesis to obtain $g(\varphi) \subseteq m\left(\varphi_{\varepsilon}\right)$. Though in the proof we use $\pi$ to ensure that $R^{d_{t}}\left(w_{t-1}, w_{t}\right)$ for $1 \leq t \leq n$, this is not strictly necessary, since if it fails, $w \in g(\rho)$ holds vacuously.

Here is an example to show that the result can fail if $\pi$ is omitted. Let $k=1$ and take

$$
\chi=\forall i(\square(j \rightarrow \diamond i) \rightarrow i \vee \diamond i \vee \diamond \diamond i),
$$

evaluated in the frame $(\{0,1,2\}, R)$, with $R=\{(0,1),(1,2)\}$. Let $h$ be a hybrid assignment with $h(j)=\{2\}$; let $p \in \mathcal{V}$ be an atom; let $m$ be a modal assignment with $m(p)=\{2\}$; and take any $\delta$ with $\operatorname{dom} \delta_{i}=\{p, \neg p\}, \operatorname{dom} \delta_{j}=\{p\}$, and $\delta_{j}(p)=1$. Then the hypothesis $h(x) \subseteq m\left(x_{\delta}\right)$ holds for all $x \in$ free $\chi$, since $h(j)=\{2\} \subseteq m\left(j_{\delta}\right)=m(p)=\{2\}$. Also, $0 \in h(\chi)$, since plainly $0 \in g(i \vee \diamond i \vee \diamond \diamond i)$ for any hybrid assignment $g$. But if $\varepsilon={ }_{i} \delta$ is such that $\varepsilon_{i}(p)=\varepsilon_{i}(\neg p)=1$, then $0 \in m\left(\square(j \rightarrow \diamond i)_{\varepsilon}\right)$ vacuously, since $m\left(j_{\varepsilon}\right)=\{2\}$ and 2 is not $R$-accessible from 0 . But $0 \notin m\left((i \vee \diamond i \vee \diamond \diamond i)_{\varepsilon}\right)$, since $i_{\varepsilon}=p \wedge \neg p$ and so $m\left(i_{\varepsilon}\right)=\varnothing$. So $0 \notin m\left(\chi_{\delta}\right)$. Hence, $h(\chi) \nsubseteq m\left(\chi_{\delta}\right)$ in this case. We leave it to the reader to check that the result can also fail for formulas of the form $\chi=\forall i_{1}, \ldots, i_{k}\left(\pi^{\diamond}\left(i_{1}, \ldots, i_{k}\right) \rightarrow \varphi\right)$.

The proof would also break down if we were to allow unrelativized $\forall$ in quasipositive formulas. For example, if $|W|=1$, then for any $h, m$ and nontrivial $\xi$, we must have $h(\forall i i)=W$, but $m\left((\forall i i)_{\xi}\right)=\varnothing$. So the soundness part of the proposition would fail.

5.4 Soundness and completeness The following are easy corollaries of Proposition 5.9.

Proposition 5.12 (Soundness) Let $\varphi$ be a clean quasi-positive sentence. If $\varphi$ is valid in $\mathcal{F}$, then so is any approximant $\varphi_{\xi}$.

Proof Let a finitary display $\xi$ be given and let $m$ be any modal assignment. Select any hybrid assignment $h$ into $\mathcal{F}$ satisfying $h(a) \subseteq m\left(a_{\xi}\right)$ for all atoms $a$. By assumption, $h(\varphi)=W$. By Proposition 5.9, $h(\varphi) \subseteq m\left(\varphi_{\xi}\right)$. The result follows.

For nonsentences, we cannot always choose such an $h$, and indeed the result can fail. For example, if $|W|=1$, then $i$ is valid in $\mathcal{F}$, but not all its approximants are.

Proposition 5.13 (Hybrid d-persistence) Let $\varphi$ be a clean quasi-positive formula, and suppose that $m: \mathcal{V} \rightarrow \wp(W)$ is a descriptive assignment into $\mathcal{F}$. Assume that for every full display $\eta$, each formula in the set $\varphi^{\eta}$ of approximants is valid in $\mathcal{F}$ under the assignment $m$; that is, $m\left(\varphi^{\eta}\right)=W$. Then $\varphi$ is valid in $\mathcal{F}$.

Proof Let $h$ be an arbitrary hybrid assignment into $\mathcal{F}$. Using Lemma 5.2, or otherwise, we may choose a full display $\eta$ with the property that $m\left(x^{\eta}\right) \subseteq h(x)$ for each $x \in$ free $\varphi$. By assumption, $m\left(\varphi^{\eta}\right)=W$. By Proposition 5.9, $m\left(\varphi^{\eta}\right) \subseteq h(\varphi)$. The result follows.

"Local" versions of these results are easily proved in the same way. For example, given $\mathcal{F}=(W, R)$, a descriptive assignment $m$ into $\mathcal{F}$, and $w \in W$, if each approximant of a quasi-positive formula $\varphi$ is true at $w$ in $\mathscr{F}$ under $h$, then $\varphi$ is also true at $w$ in all models with frame $\mathcal{F}$. 
Definition 5.14 If $\Phi$ is a set of quasi-positive sentences, we write $\Lambda_{\Phi}$ for the normal modal logic axiomatized by the set of axioms

$$
\bigcup\left\{\varphi^{\eta}: \varphi \in \Phi, \eta \text { a full display }\right\}=\left\{\varphi_{\delta}: \varphi \in \Phi, \delta \text { a finitary display }\right\} .
$$

For a single quasi-positive sentence $\varphi$, we write $\Lambda_{\varphi}$ instead of $\Lambda_{\{\varphi\}}$.

Theorem 5.15 Let $\Phi$ be a set of clean quasi-positive sentences. Then $\Lambda_{\Phi}$ is canonical, its canonical frame validates $\Phi$, and $\Lambda_{\Phi}$ is sound and complete for the class of frames validating all $\varphi \in \Phi$.

Proof Each approximant in $\varphi^{\eta}$, for each $\varphi \in \Phi$ and each full display $\eta$, is valid in the canonical model for $\Lambda_{\Phi}$. The canonical assignment is well known to be descriptive, so by Proposition 5.13, each $\varphi \in \Phi$ is valid in the canonical frame $g$ for $\Lambda_{\Phi}$. Hence, by Proposition 5.12, each axiom of $\Lambda_{\Phi}$ is also valid in $g$, and so every formula in $\Lambda_{\Phi}$ is valid in $g$. This establishes canonicity of $\Lambda_{\Phi}$. It also follows that any nontheorem of $\Lambda_{\Phi}$ can be invalidated on a frame validating $\Phi$, namely, $g$. Conversely, by Proposition 5.12, all the axioms of $\Lambda_{\Phi}$ are valid in frames validating all formulas in $\Phi$. Hence, all theorems of $\Lambda_{\Phi}$ are valid in these frames. So, indeed, $\Lambda_{\Phi}$ is the modal logic of the class of frames validating $\Phi$.

We stated Theorem 5.15 for quasi-positive sentences, but there is no loss of strength if we restrict it to pure quasi-positive sentences. For it is easily seen that any quasipositive sentence $\varphi\left(q_{1}, \ldots, q_{n}\right)$ is valid in the same frames as $\varphi(\perp, \ldots, \perp)$, which is pure. Similarly, $\Lambda_{\varphi\left(q_{1}, \ldots, q_{n}\right)}$ has the same frames as $\Lambda_{\varphi(\perp, \ldots, \perp) \text {. }}$.

5.5 Elementarily generated modal logics A modal logic is said to be elementarily generated if it is the modal logic of some elementary class of frames. By Fine's theorem [7], all such logics are canonical. The converse fails in general, as was shown in [17] and [16], but the elementarily generated logics still form an important class of well-behaved logics, and in practice, the usual way to show that a modal logic is canonical is by establishing that it is elementarily generated. We can now characterize the elementarily generated modal logics by the following theorem.

Theorem 5.16 The elementarily generated modal logics are precisely the ones of the form $\Lambda_{\Phi}$, for some set $\Phi$ of pure clean quasi-positive hybrid sentences.

Proof The right-to-left direction is immediate-given $\Phi$ as in the theorem, $\Lambda_{\Phi}$ is the modal logic of the class of frames in which every sentence in $\Phi$ is valid, and this class is elementary. Conversely, let $\Lambda$ be the modal logic of some elementary class $\mathcal{K}$ of frames. Let $\Psi_{\mathcal{K}}$ be the set of all pseudo-equational $L$-sentences true in each frame in $\mathcal{K}$. By [12], Theorem 4.13, $\Lambda$ is also the modal logic of the class $\overline{\mathcal{K}}=\left\{\mathcal{F}: \mathcal{F} \models \Psi_{\mathcal{K}}\right\} .{ }^{5}$ By Theorem 3.13, there is a set $\Phi$ of pure quasi-positive sentences such that $\overline{\mathcal{K}}$ is the class of frames that validate every $\varphi \in \Phi$. By Remark 5.4, we can assume that the sentences in $\Phi$ are clean-in fact, Theorem 3.13 can be set up to produce clean sentences. By Theorem 5.15, $\Lambda=\Lambda_{\Phi}$.

\section{Remarks and Problems}

In this paper, we have shown how to effectively axiomatize any elementarily generated modal logic. We end with some discussion about the work presented here. 
6.1 Canonicity We can deduce from Theorem 5.15 the known facts that the McKinsey-Lemmon logic (discussed in Example 4.6) and Hughes's logic (Example 4.7) are canonical. Further, it is perhaps worth mentioning that our results provide a new proof of Fine's theorem.

Corollary 6.1 (Fine, [7]) Any elementarily generated modal logic is canonical.

Proof By Theorems 5.15 and 5.16.

6.2 The axioms If, in the proof of Theorem 5.16, there is a recursively enumerable first-order axiomatization of $\mathcal{K}$, then $\Psi_{\mathcal{K}}, \Phi$, and $\Lambda_{\Phi}$ are all recursively enumerable. ${ }^{6}$ If $\mathcal{K}$ is already axiomatized by explicit pseudo-equations, then we can construct $\Phi$ and the axioms for $\Lambda_{\Phi}$ effectively and explicitly-we actually get to see the axioms, and they can be fairly transparent. For example, the axioms we obtain for the logics $K M^{\infty}$ and $K M T$ coincide (modulo standard equivalences) with the existing axiomatizations of these logics given in [30] and [24], respectively.

It is possible to extend Propositions 5.9, 5.12, 5.13, and Theorem 5.15 from quasipositive sentences to "hybrid Sahlqvist formulas" as defined in Example 3.6. However, it turns out that for any such formula there is an equivalent pure quasi-positive sentence. So this gives no increase in expressivity and no additional logics that can be axiomatized by approximants. For this reason, we do not include the generalization in this paper; it can be an exercise for the interested reader. However, it is perhaps noteworthy that the axioms obtained as approximants to a hybrid Sahlqvist formula can be simpler than the approximants of the corresponding quasi-positive sentence, and indeed, the approximants of a modal Sahlqvist formula are simply its substitution instances. Accepting this, we see that the generalized Theorem 5.15 would imply the completeness part of Sahlqvist's theorem.

At present, the methods presented here do not seem to shed light on when $\Lambda_{\Phi}$ is finitely axiomatizable. The set of axioms we construct for $\Lambda_{\Phi}$ is always infinite, even when $\Phi$ is finite, because a quasi-positive hybrid sentence has infinitely many approximants. But sometimes the blowup occurs when obtaining $\Phi$ itself, as Example 6.3 below shows.

Proposition 6.2 Let $\varphi$ be any pure clean quasi-positive sentence. Then a finite frame is a frame for $\Lambda_{\varphi}$ if and only if it validates $\varphi$. Hence, it is decidable whether a finite frame validates $\Lambda_{\varphi}$.

Proof Let $\mathcal{F}=(W, R)$ be a finite frame. By Proposition 5.12, if $\varphi$ is valid in $\mathcal{F}$ then so is $\Lambda_{\varphi}$. Conversely, assume that $\Lambda_{\varphi}$ is valid in $\mathcal{F}$. Let $m: \mathcal{V} \rightarrow \wp(W)$ be any surjective map. It is easily checked that $m$ is a descriptive assignment into $\mathcal{F}$. By assumption, $m\left(\varphi^{\eta}\right)=W$ for every full display $\eta$. By Proposition 5.13, $\varphi$ is valid in $\mathcal{F}$.

Hence we may test for $\Lambda_{\varphi}$ being valid in a finite frame $\mathcal{F}$ by evaluating $\varphi$ in $\mathcal{F}$; this can be done by an algorithm.

Example 6.3 $K^{3}$ is the logic of the class of products of three Kripke frames (see [8] for information). $K^{3}$ is not finitely axiomatizable, but it is the logic of a finitely axiomatizable elementary class of frames [29]. By Theorem 5.16 and the comments above, $K^{3}=\Lambda_{\Phi}$ for some set $\Phi$ of pure quasi-positive sentences, and $K^{3}$ is recursively enumerable. However, it follows from Proposition 6.2 that $K^{3}$ is not of 
the form $\Lambda_{\varphi}$ for any pure quasi-positive sentence $\varphi$, since by [20], it is undecidable whether a finite frame is a frame for this logic.

Taking an algebraic view for a moment, the same argument shows that the variety RRA of representable relation algebras cannot be axiomatized by the approximants of any single pure quasi-positive sentence, since it is undecidable whether a finite relation algebra is representable [21]. As with $K^{3}$, there is a finitely axiomatizable elementary class of atom structures (e.g., the class of atom structures of full relation set algebras) whose complex algebras generate RRA.

6.3 Correspondence Here we comment on the relation between the class $\operatorname{Fr} \varphi$ of frames validating a pure quasi-positive sentence $\varphi$, and $\operatorname{Fr} \Lambda_{\varphi}$, the class of frames that validate the approximants of $\varphi$. That $\operatorname{Fr} \varphi \subseteq \operatorname{Fr} \Lambda_{\varphi}$ is clear, by Theorem 5.15.

Though by Proposition 6.2 it is true in finite frames, it is false in general that if $\varphi$ is a quasi-positive sentence whose approximants are valid in a frame $\mathcal{F}$, then $\varphi$ itself is valid in $\mathcal{F}$. That is, the inclusion $\operatorname{Fr} \varphi \subseteq \operatorname{Fr} \Lambda_{\varphi}$ may be strict. This behavior is a notable difference from the classical Sahlqvist theorem. There, the "approximants" of a Sahlqvist formula $\sigma$ are just the substitution instances of $\sigma$, and these include $\sigma$ itself. If the approximants are valid in a frame $\mathcal{F}$, then so is $\sigma$, and so the first-order correspondent of $\sigma$ is true in $\mathcal{F}$. So the frames for $\sigma$ are precisely the frames for its approximants, and they form an elementary class.

If $\varphi$ is equivalent to the correspondent of a Sahlqvist formula $\sigma$, then we do have $\operatorname{Fr} \varphi=\operatorname{Fr} \Lambda_{\varphi}$. This is because $\sigma$ is valid in all frames in $\operatorname{Fr} \varphi$, and so $\sigma \in \Lambda_{\varphi}$ by Theorem 5.15. Hence, $\sigma$ and its correspondent $\varphi$ are valid in all frames in $\operatorname{Fr} \Lambda_{\varphi}$.

In general, however, $\operatorname{Fr} \varphi$, which is elementary and finitely axiomatizable, may be properly contained in $\operatorname{Fr} \Lambda_{\varphi}$. We will give two examples of this. In the first, $\operatorname{Fr} \Lambda_{\varphi}$ is also elementary and finitely axiomatizable and of the form $\operatorname{Fr} \psi$ for a (rather weaker) quasi-positive sentence $\psi$. In the second, $\operatorname{Fr} \Lambda_{\varphi}$ is nonelementary.

Example 6.4 This is based on [39], Example 2.4. Consider the following pure quasi-positive sentences:

$$
\begin{aligned}
& \varphi=\exists j(\diamond j \wedge \forall i(\square(j \rightarrow \diamond i) \rightarrow \square \diamond i)), \\
& \psi=\forall i(\diamond i \rightarrow \forall j(\diamond j \rightarrow \exists k(\diamond k \wedge \forall l[\square(k \rightarrow \diamond l) \rightarrow \diamond(i \wedge \diamond l) \wedge \diamond(j \wedge \diamond l)]))) .
\end{aligned}
$$

Given a frame $\mathcal{F}=(W, R)$ and $w \in W$, write $R^{w}$ for $\{v \in W: R(w, v)\}$. Then

1. $\mathcal{F}, w \models \varphi$ iff $\left\{R^{x}: x \in R^{w}\right\}$ contains a $\subseteq$-minimum element,

2. $\mathcal{F}, w \models \psi$ iff $\left\{R^{x}: x \in R^{w}\right\}$ is $\supseteq$-directed: any two sets in it contain a third.

Clearly, $\operatorname{Fr} \varphi \subseteq \operatorname{Fr} \psi$. However, $(\mathbb{N},<) \in \operatorname{Fr} \psi \backslash \operatorname{Fr} \varphi$, so the inclusion is strict.

[39] shows that the modal logics of $\operatorname{Fr} \varphi$ and $\operatorname{Fr} \psi$ (i.e., $\Lambda_{\varphi}$ and $\Lambda_{\psi}$, respectively) are the same and are finitely axiomatized by the Sahlqvist formula

$$
\sigma=\diamond \square p \wedge \diamond \square q \rightarrow \diamond \square(p \wedge q),
$$

expressing that the formula $\square \diamond p$ is additive in $p$. On the one hand, $\psi$ is equivalent to the first-order correspondent of $\sigma$, so $\operatorname{Fr} \psi$ is the class of all frames for $\sigma$, and $\operatorname{Fr} \psi=\operatorname{Fr} \Lambda_{\psi}$. On the other hand, $\varphi$ is equivalent to the "canonical pseudocorrespondent" of $\sigma$ obtained in [39]: $\mathcal{F}=\varphi \Rightarrow \mathcal{F} \models \sigma$ for any frame $\mathcal{F}$, and the canonical frame of the logic axiomatized by $\sigma$ validates $\varphi .^{7}$ This shows that

1. there are inequivalent $\varphi, \psi$ such that $\Lambda_{\varphi}=\Lambda_{\psi}$,

2. there is $\varphi$ such that $\operatorname{Fr} \Lambda_{\varphi} \supset \operatorname{Fr} \varphi$, and yet $\operatorname{Fr} \Lambda_{\varphi}$ is elementary and finitely axiomatizable. 
Example 6.5 Consider $\varphi=\diamond \exists i(i \wedge \diamond i)$. This is the formula considered in Example 4.7 in relation to Hughes's logic $K M T$ [24]. It is valid in precisely the frames such that every world sees a reflexive world. It is therefore not valid in the frame $\mathcal{N}=(\mathbb{N}, \neq)$ (i.e., the complete graph on $\mathbb{N})$.

Now in [24], Theorem 12, it is shown that the frames for KMT (by Example 4.7, these are the frames validating $\Lambda_{\varphi}$ ) are precisely those frames such that the graph based on the successors of each world is not finitely colorable in the sense of graph theory. More formally, for each world $x$, the set of worlds accessible from $x$ cannot be partitioned into finitely many sets such that no world in any partition set can see a world in the same set. In $\mathcal{N}$, the graph on the successors of any point in $(\mathbb{N}, \neq)$ is a complete infinite graph and so cannot be finitely colored. Hence, $\mathcal{N}$ is a frame for $K M T$, and so all approximants of $\varphi$ are valid in $\mathcal{N}$. (For example, the approximant $\alpha=\diamond((q \wedge \diamond q) \vee(\neg q \wedge \diamond \neg q))$ is certainly valid in $\mathcal{N}$, since either $q$ or $\neg q$ must be assigned to an infinite subset of $\mathbb{N}$, and any point in such a set will make the corresponding disjunct in $\alpha$ true.)

We conclude that $\mathcal{N} \in \operatorname{Fr} \Lambda_{\varphi} \backslash \operatorname{Fr} \varphi$, and hence $\operatorname{Fr} \Lambda_{\varphi} \supset \operatorname{Fr} \varphi$. Indeed, an ultraproduct argument similar to that in [22], Corollary 7, will show that the class $\operatorname{Fr} \Lambda_{\varphi}$ of frames for $K M T$ is not elementary.

6.4 Irreflexivity Following on from Example 3.9, we can now see that the irreflexivity axiom $\exists i(i \wedge \square \neg i)$ is not equivalent to a pure quasi-positive sentence. For suppose that it were equivalent to such a sentence, say $\varphi$. Then by Theorem 5.15 (soundness), $\Lambda_{\varphi}$ is valid in the irreflexive frame $(\mathbb{Z},<)$. Define $m: \mathcal{V} \rightarrow \wp(\mathbb{Z})$ by $m(q)=\varnothing$ for all $q$. Then, clearly, $\Gamma=\{\alpha \in \mathscr{L}:(\mathbb{Z},<), m, w \models \alpha\}$ is independent of $w \in \mathbb{Z}$ and is a maximal $\Lambda_{\varphi}$-consistent set. It is easily seen that $\Gamma$ is a reflexive point in the canonical frame for $\Lambda_{\varphi}$ which therefore does not validate $\varphi$. This contradicts Theorem 5.15. An alternative proof uses suitable bounded morphisms.

\subsection{Problems}

Problem 6.6 Let $\Phi$ be a set of pure quasi-positive sentences. Consider the properties,

(a) $\operatorname{Fr} \Lambda_{\Phi}=\operatorname{Fr} \Phi$,

(b) $\operatorname{Fr} \Lambda_{\Phi}$ is elementary and finitely axiomatizable,

(c) $\operatorname{Fr} \Lambda_{\Phi}$ is elementary,

(d) $\Lambda_{\Phi}$ is finitely axiomatizable,

(e) $\Lambda_{\Phi}$ has a canonical axiomatization,

(f) $\Lambda_{\Phi}$ is axiomatizable by canonical formulas plus finitely many additional axioms.

We have (a) $\vee(\mathrm{b}) \Rightarrow(\mathrm{c})$, and (d) $\Rightarrow(\mathrm{e}) \Rightarrow(\mathrm{f})$. Also, (c) $\wedge(\mathrm{d}) \Rightarrow(\mathrm{b})$, because (c) and (d) imply that $\operatorname{Fr} \Lambda_{\Phi}$ and its complement are both closed under ultraproducts. If (c) holds, then $\operatorname{Fr} \Lambda_{\Phi}=\operatorname{Fr} \Lambda_{\Psi}=\operatorname{Fr} \Psi$ for some set $\Psi$ of pure quasi-positive sentences (namely, those valid in $\operatorname{Fr} \Lambda_{\Phi}$ ). The converse is trivially true. By the same argument plus first-order compactness, (b) is equivalent to saying that $\operatorname{Fr} \Lambda_{\Phi}=\operatorname{Fr} \Lambda_{\psi}=\operatorname{Fr} \psi$ for some pure quasi-positive sentence $\psi$. If $\Phi$ is finite, then (a) $\Rightarrow$ (b); the converse fails by Example 6.4. $K M^{\infty}$ and $K M T$ lack all six properties even though their " $\Phi$ " is finite. 
What, if any, other implications are there among these properties? Characterize the $\Phi$ for which each of the properties holds. (Restrict attention to finite $\Phi$ if it helps.)

Problem 6.7 What can be said about the first-order theory of the canonical frame for $\Lambda_{\Phi}$ ?

Problem 6.8 What more can be said about a logic $\Lambda_{\varphi}$ by studying the form of $\varphi$ ? For example, can decidability and complexity results be obtained? What can be said when restrictions are imposed on $\varphi$-for example, that it has no universal quantifiers?

Problem 6.9 Generalize the results to the algebraic setting: Boolean algebras with operators, lattices. In this vein, when are logics $\Lambda_{\Phi}$ preserved under completions? See [9].

Problem 6.10 Are there consequences of our results for hybrid logic itself?

\section{Notes}

1. (§1.1) Related results were proved later by van Benthem ([36], [37]) and Goldblatt ([10], [12]). There is a useful discussion in [14], §6.6.

2. (after (1) in §1.3) [3] uses the condition $\forall x \exists y(R(x, y) \wedge \forall z(R(y, z) \rightarrow z=y))$; in transitive frames, this is equivalent to (1).

3. (§1.3) Or more generally, any locally elementary and locally d-persistent formula.

4. (after Definition 3.11) Pseudo-equations are preserved under bounded morphic images, disjoint unions, and generated subframes, and any set of first-order $L$-sentences so preserved is equivalent to a set of pseudo-equations; see [38] for binary $R$ and [10], §4, for arbitrary arities.

5. (Theorem 5.16) In [12], Goldblatt actually states the result algebraically: if $\mathcal{K}$ is closed under ultraproducts, the variety of algebras generated by $\mathcal{K}$ is the class of subalgebras of complex algebras over frames in $\operatorname{Mod} \Psi_{\mathcal{K}}$. [12] also shows that $\overline{\mathcal{K}}$ is the closure of $\mathcal{K}$ under bounded morphic images, generated subframes, disjoint unions, and ultraroots. Related results were proved by Goldblatt ([10] §4, [11], and [13]) and van Benthem [38].

6. (\$6.2) Van Benthem noted in [37], p. 138, that if a class of frames is definable by means of a recursive or recursively enumerable first-order theory, then its modal theory is recursively axiomatizable.

7. (Example 6.4) In [39], Venema shows that for any positive modal formula $\pi(p)$ involving a single atom, $p$, the additivity axiom $\pi(p \vee q) \leftrightarrow \pi(p) \vee \pi(q)$ has a canonical pseudo-correspondent. Written in $\mathscr{H}(@, \downarrow)$, it amounts to $\downarrow_{i} \neg \pi\left(\neg \downarrow j @_{i} \pi(j)\right)$. For $\pi(p)=\square \diamond p$, this is $\downarrow_{i} \diamond \square \downarrow_{j} @_{i} \square \diamond j$, which is equivalent to $\varphi$. 


\section{References}

[1] Areces, C., P. Blackburn, and M. Marx, "Hybrid logic is the bounded fragment of first order logic," pp. 33-50 in Proceedings of 6th Workshop on Logic, Language, Information and Computation, WOLLIC99, edited by R. de Queiroz and W. Carnielli, 1999. 457,458

[2] Balbiani, P., I. Shapirovsky, and V. Shehtman, "Every world can see a Sahlqvist world," pp. 69-85 in Advances in Modal Logic. Vol. 6, edited by G. Governatori, I. Hodkinson, and Y. Venema, College Publications, London, 2006. 445, 446, 450

[3] Blackburn, P., M. de Rijke, and Y. Venema, Modal Logic, vol. 53 of Cambridge Tracts in Theoretical Computer Science, Cambridge University Press, Cambridge, 2001. Zbl 0988.03006. MR 1837791. 445, 448, 450, 453, 465, 475

[4] Chagrov, A., and M. Zakharyaschev, Modal Logic, vol. 35 of Oxford Logic Guides, The Clarendon Press, New York, 1997. Zbl 0871.03007. MR 1464942. 445, 465, 466

[5] Chang, C. C., and H. J. Keisler, Model Theory, 3d edition, vol. 73 of Studies in Logic and the Foundations of Mathematics, North-Holland Publishing Co., Amsterdam, 1990. Zbl 0697.03022. MR 1059055. 466

[6] Cresswell, M. J., "A Henkin completeness for T," Notre Dame Journal of Formal Logic, vol. 8 (1967), pp. 186-90. Zbl 0183.00802. MR 0281588. 444

[7] Fine, K., "Some connections between elementary and modal logic," pp. 15-31 in Proceedings of the 3rd Scandinavian Logic Symposium (Uppsala, 1973), vol. 82 of Studies in Logic and the Foundations of Mathematics, Amsterdam, 1975. North-Holland, Zbl 0316.02021. MR 0401437. 444, 446, 448, 471, 472

[8] Gabbay, D. M., A. Kurucz, F. Wolter, and M. Zakharyaschev, Many-Dimensional Modal Logics: Theory and Applications, vol. 148 of Studies in Logic and the Foundations of Mathematics, North-Holland Publishing Co., Amsterdam, 2003. Zbl 1051.03001. MR 2011128. 472

[9] Givant, S., and Y. Venema, "The preservation of Sahlqvist equations in completions of Boolean algebras with operators," Algebra Universalis, vol. 41 (1999), pp. 47-84. Zbl 0964.03033. MR 1682042. 475

[10] Goldblatt, R., "Varieties of complex algebras," Annals of Pure and Applied Logic, vol. 44 (1989), pp. 173-242. Zbl 0722.08005. MR 1020344. 475

[11] Goldblatt, R., Mathematics of Modality, vol. 43 of CSLI Lecture Notes, Stanford University CSLI, Stanford, 1993. Zbl 0942.03516. MR 1317099. 475

[12] Goldblatt, R., "Elementary generation and canonicity for varieties of Boolean algebras with operators," Algebra Universalis, vol. 34 (1995), pp. 551-607. Zbl 0854.06020. MR 1357484. 454, 471, 475

[13] Goldblatt, R., "Quasi-modal equivalence of canonical structures," The Journal of Symbolic Logic, vol. 66 (2001), pp. 497-508. Zbl 0990.03011. MR 1833461. 475

[14] Goldblatt, R., "Mathematical modal logic: A view of its evolution," pp. 1-98 in Handbook of the History of Logic, Vol. 7: Logic and the Modalities in the Twentieth Century, edited by D. Gabbay and J. Woods, Elsevier, 2006. Originally published in Journal of Applied Logic, vol. 1 (2003), pp. 309-92. Zbl 1041.03015. MR 2021314. 475 
[15] Goldblatt, R., and I. Hodkinson, "The McKinsey-Lemmon logic is barely canonical," forthcoming in Australasian Journal of Logic, (2006).

http://www.philosophy.unimelb.edu.au/aj1/. 445, 450

[16] Goldblatt, R., I. Hodkinson, and Y. Venema, "On canonical modal logics that are not elementarily determined," Logique et Analyse. Nouvelle Série, vol. 46 (2003), pp. 77101. Zbl 1060.03037. MR 2102338. 444, 471

[17] Goldblatt, R., I. Hodkinson, and Y. Venema, "Erdôs graphs resolve Fine's canonicity problem,” The Bulletin of Symbolic Logic, vol. 10 (2004), pp. 186-208. Zbl 1060.03038. MR 2062417. 444, 471

[18] Goranko, V., and D. Vakarelov, "Sahlqvist formulas in hybrid polyadic modal logics," Journal of Logic and Computation, vol. 11 (2001), pp. 737-54. Hybrid logics (Birmingham, 2000). Zbl 0993.03013. MR 1866657. 445, 449

[19] Goranko, V., and D. Vakarelov, "Elementary canonical formulae: Extending Sahlqvist's theorem," Annals of Pure and Applied Logic, vol. 141 (2006), pp. 180-217. Zbl 05047224. MR 2229935. 445, 466

[20] Hirsch, R., I. Hodkinson, and A. Kurucz, "On modal logics between $\mathbf{K} \times \mathbf{K} \times \mathbf{K}$ and S5 $\times$ S5 $\times$ S5," The Journal of Symbolic Logic, vol. 67 (2002), pp. 221-34. Zbl 1002.03019. MR 1889547. 473

[21] Hirsch, R., and I. Hodkinson, "Representability is not decidable for finite relation algebras," Transactions of the American Mathematical Society, vol. 353 (2001), pp. 1403-25 (electronic). Zbl 0965.03079. MR 1806735. 473

[22] Hirsch, R., and I. Hodkinson, "Strongly representable atom structures of relation algebras," Proceedings of the American Mathematical Society, vol. 130 (2002), pp. 1819-31 (electronic). Zbl 1002.03054. MR 1887031. 450, 474

[23] Hodkinson, I., and Y. Venema, "Canonical varieties with no canonical axiomatisation," Transactions of the American Mathematical Society, vol. 357 (2005), pp. 4579-4605 (electronic). Zbl 1081.03062. MR 2156722. 446, 461

[24] Hughes, G. E., "Every world can see a reflexive world," Studia Logica, vol. 49 (1990), pp. 175-81. Zbl 0719.03010. MR 1094537. 445, 461, 472, 474

[25] Jónsson, B., “On the canonicity of Sahlqvist identities,” Studia Logica, vol. 53 (1994), pp. 473-91. Zbl 0810.03050. MR 1309894. 446

[26] Jónsson, B., and A. Tarski, "Boolean algebras with operators. I," American Journal of Mathematics, vol. 73 (1951), pp. 891-939. Zbl 0045.31505. MR 0044502. 444, 449

[27] Kikot, S., "A new generalization of Sahlqvist theorem,” Preprint, 2005. 445

[28] Kracht, M., Tools and Techniques in Modal Logic, vol. 142 of Studies in Logic and the Foundations of Mathematics, North-Holland Publishing Co., Amsterdam, 1999. Zbl 0927.03002. MR 1707315. 445

[29] Kurucz, Á., “On axiomatising products of Kripke frames," The Journal of Symbolic Logic, vol. 65 (2000), pp. 923-45. Zbl 0963.03027. MR 1771094. 472 
[30] Lemmon, E. J., An Introduction to Modal Logic, edited by K. Segerberg, vol. 11 of American Philosophical Quarterly, Monograph Series, Basil Blackwell, Oxford, 1977. The "Lemmon Notes," in collaboration with Dana Scott. Zbl 0388.03006. MR 0476365. 444, 445, 460, 472

[31] Makinson, D., “On some completeness theorems in modal logic," Zeitschrift für mathematische Logik und Grundlagen der Mathematik, vol. 12 (1966), pp. 379-84. Zbl 0295.02014. MR 0223220. 444

[32] Sahlqvist, H., "Completeness and correspondence in the first and second order semantics for modal logic," pp. 110-43 in Proceedings of the 3rd Scandinavian Logic Symposium (Uppsala, 1973), vol. 82 of Studies in Logic and the Foundations of Mathematics, NorthHolland, Amsterdam, 1975. Zbl 0319.02018. MR 0387008. 444, 448

[33] Sambin, G., and V. Vaccaro, "A new proof of Sahlqvist's theorem on modal definability and completeness," The Journal of Symbolic Logic, vol. 54 (1989), pp. 992-99. Zbl 0682.03009. MR 1011185. 448, 462

[34] ten Cate, B., M. Marx, and P. Viana, "Hybrid logics with Sahlqvist axioms," Logic Journal of the IGPL, vol. 13 (2005), pp. 293-300. Zbl 1080.03006. MR 2157422. 445, 449

[35] Vakarelov, D., "Modal definability in languages with a finite number of propositional variables and a new extension of the Sahlqvist's class," pp. 499-518 in Advances in Modal Logic. Vol. 4, edited by P. Balbiani, N-Y. Suzuki, F. Wolter, and M. Zakharyaschev, King's College Publications, London, 2003. Zbl 1081.03018. MR 2112097. 445

[36] van Benthem, J. F. A. K., "Canonical modal logics and ultrafilter extensions," The Journal of Symbolic Logic, vol. 44 (1979), pp. 1-8. Zbl 0405.03011. MR 523481. 475

[37] van Benthem, J. F. A. K., "Some kinds of modal completeness," Studia Logica, vol. 39 (1980), pp. 125-41. Zbl 0459.03008. MR 595108. 475

[38] van Benthem, J., Modal Logic and Classical Logic, Indices: Monographs in Philosophical Logic and Formal Linguistics, III. Bibliopolis, Naples, 1985. Zbl 0639.03014. MR 832432. 475

[39] Venema, Y., "Canonical pseudo-correspondence," pp. 421-30 in Advances in Modal Logic. Vol. 2 (Uppsala, 1998), edited by M. Zakharyaschev, K. Segerberg, M. de Rijke, and H. Wansing, vol. 119 of CSLI Lecture Notes, CSLI Publications, Stanford, 2001. Zbl 0993.03022. MR 1838261. 446, 447, 450, 461, 473, 475

\section{Acknowledgments}

I thank Robert Goldblatt and the members of the London Logic Forum for helpful discussions and encouragement. (http://llf.dcs.kcl.ac.uk/)

Department of Computing

Imperial College London

London SW7 2AZ

UNITED KINGDOM

imh@doc.ic.ac.uk

http://www.doc.ic.ac.uk/ imh 